\title{
UNCERTAINTIES IN THE $v p$-PROCESS: SUPERNOVA DYNAMICS VERSUS NUCLEAR PHYSICS
}

\author{
Shinya Wanajo ${ }^{1,2}$, Hans-Thomas Janka ${ }^{2}$, AND Shigeru Kubono ${ }^{3}$ \\ ${ }^{1}$ Technische Universität München, Excellence Cluster Universe, Boltzmannstr. 2, D-85748 Garching, Germany; shinya.wanajo@ universe-cluster.de \\ ${ }^{2}$ Max-Planck-Institut für Astrophysik, Karl-Schwarzschild-Str. 1, D-85748 Garching, Germany; thj@ mpa-garching.mpg.de \\ ${ }^{3}$ Center for Nuclear Study, University of Tokyo, RIKEN Campus, 2-1 Hirosawa, Wako, Saitama 351-0198, Japan; kubono@ cns.s.u-tokyo.ac.jp \\ Received 2010 April 14; accepted 2010 December 27; published 2011 February 8
}

\begin{abstract}
We examine how the uncertainties involved in supernova dynamics, as well as in nuclear data inputs, affect the $v p$-process in the neutrino-driven winds. For the supernova dynamics, we find that the wind termination by the preceding dense ejecta shell, as well as the electron fraction $\left(Y_{\mathrm{e}, 3} ;\right.$ at $\left.3 \times 10^{9} \mathrm{~K}\right)$, plays a crucial role. A wind termination within the temperature range of $(1.5-3) \times 10^{9} \mathrm{~K}$ greatly enhances the efficiency of the $v p$-process. This implies that the early wind phase, when the innermost layer of the preceding supernova ejecta is still $\sim 200-1000 \mathrm{~km}$ from the center, is most relevant to the $v p$-process. The outflows with $Y_{\mathrm{e}, 3}=0.52-0.60$ result in the production of the $p$-nuclei up to $A=108$ with interesting amounts. Furthermore, the $p$-nuclei up to $A=152$ can be produced if $Y_{\mathrm{e}, 3}=0.65$ is achieved. For the nuclear data inputs, we test the sensitivity to the rates relevant to the breakout from the $p-p$ chain region $(A<12)$, to the $(n, p)$ rates on heavy nuclei, and to the nuclear masses along the $v p$-process pathway. We find that a small variation of the rates of triple- $\alpha$ and of the $(n, p)$ reaction on ${ }^{56} \mathrm{Ni}$ leads to a substantial change in the $p$-nuclei production. We also find that ${ }^{96} \mathrm{Pd}(N=50)$ on the $v p$-process path plays a role as a second seed nucleus for the production of heavier $p$-nuclei. The uncertainty in the nuclear mass of ${ }^{82} \mathrm{Zr}$ can lead to a factor of two reduction in the abundance of the $p$-isotope ${ }^{84} \mathrm{Sr}$.
\end{abstract}

Key words: nuclear reactions, nucleosynthesis, abundances - stars: abundances - stars: neutron - supernovae: general

Online-only material: color figures

\section{INTRODUCTION}

The astrophysical origin of the proton-rich isotopes of heavy elements ( $p$-nuclei) is not fully understood. The most successful model to date, the photodissociation of pre-existing neutron-rich isotopes ( $\gamma$-process) in the oxygen-neon layer of core-collapse supernovae (or in their pre-collapse stages), cannot explain the production of some light $p$-nuclei including ${ }^{92,94} \mathrm{Mo}$ and ${ }^{96,98} \mathrm{Ru}$ (Woosley \& Howard 1978; Prantzos et al. 1990; Rayet et al. 1995; Rauscher et al. 2002; Hayakawa et al. 2008). The recent discovery of a new nucleosynthetic process, the $v p$-process, has dramatically changed this difficult situation (Fröhlich et al. 2006a, 2006b; Pruet et al. 2006; Wanajo 2006). In the early neutrino-driven winds of core-collapse supernovae, $\bar{v}_{e}$ capture on free protons gives rise to a tiny amount of free neutrons in the proton-rich matter. These neutrons induce the $(n, p)$ reactions on the $\beta^{+}$-waiting point nuclei along the classical rp-process path $\left({ }^{64} \mathrm{Ge},{ }^{68} \mathrm{Se}\right.$, and ${ }^{72} \mathrm{Kr}$ ), which bypass these nuclei (with the $\beta^{+}$-decay half-lives of 1.06 minutes, $35.5 \mathrm{~s}$, and $17.1 \mathrm{~s}$, respectively). Wanajo (2006) has shown that the $p$-nuclei up to $A \sim 110$, including ${ }^{92,94} \mathrm{Mo}$ and ${ }^{96,98} \mathrm{Ru}$, can be produced by the $v p$-process in the neutrino-driven winds within reasonable ranges of the model parameters.

All the recent hydrodynamic studies of core-collapse supernovae with neutrino transport taken into account suggest that the bulk of early supernova ejecta is proton rich (Janka et al. 2003; Liebendörfer et al. 2003; Buras et al. 2006; Kitaura et al. 2006; Fischer et al. 2010; Hüdepohl et al. 2010). This supports the $v p$-process taking place in the neutrino-driven winds of core-collapse supernovae. However, different works end up with somewhat different outcomes. Fröhlich et al. (2006a) showed that the $p$-nuclei up to $A \sim 80$ were produced with the onedimensional, artificially induced explosion model of a $20 M_{\odot}$ star, while Pruet et al. (2006) obtained up to $A \sim 100$ with the two-dimensional, artificially induced explosion model of a $15 M_{\odot}$ star. In contrast, Wanajo et al. (2009; also S. Wanajo et al. 2011, in preparation) found negligible contribution of the $v p$-process to the production of $p$-nuclei with the onedimensional, self-consistently exploding model of a $9 M_{\odot}$ star (electron-capture supernova; Kitaura et al. 2006; Hüdepohl et al. 2010). These diverse outcomes indicate that the $v p$-process is highly sensitive to the physical conditions of neutrino-driven winds.

Besides the supernova conditions, there could also be uncertainties in some key nuclear rates, in particular of $(n, p)$ reactions, because no attention was paid to neutron-capture reactions on proton-rich nuclei before the discovery of the $v p$-process. Uncertainties in some reactions relevant to the breakout from the $p-p$ chain region $(A<12)$, which affect the proton-to-seed ratio at the onset of $v p$-processing, might also influence the nucleosynthetic outcomes. There are still a number of isotopes without experimental nuclear masses on the $v p$-process pathway (Weber et al. 2008).

Our goal in this paper is to examine how the variations of supernova conditions, as well as of nuclear data inputs, influence the global trend of the $v p$-process. This paper is organized as follows. In Section 2, a basic picture of the $v p$-process is outlined. A semi-analytic neutrino-driven wind model and an up-to-date reaction network code are described, which are used in this study (Section 3). We take the wind-termination radius (or temperature), the neutrino luminosity, the neutronstar mass, and the electron fraction as the key parameters of supernova conditions (Section 4). In previous studies (Fröhlich et al. 2006a, 2006b; Pruet et al. 2006; Wanajo 2006), some of these parameters were varied to test their sensitivities, but only for limited cases. In particular, the effect of wind termination 
has not been discussed at all in previous studies. As the key nuclear reactions, we take triple- $\alpha,{ }^{7} \mathrm{Be}(\alpha, \gamma){ }^{11} \mathrm{C},{ }^{10} \mathrm{~B}(\alpha, p){ }^{13} \mathrm{C}$ (all relevant to the breakout from the $p-p$ chain region), and the $(n, p)$ reactions on ${ }^{56} \mathrm{Ni},{ }^{60} \mathrm{Zn}$, and ${ }^{64} \mathrm{Ge}$ (Section 5). Sensitivities of the masses of the nuclei along the $v p$-process path are also discussed. We then discuss the possible role of the $v p$-process as the astrophysical origin of the $p$-nuclei (Section 6). A summary of our results follows in Section 7.

\section{BASIC PICTURE OF THE $v p$-PROCESS}

The " $v p$-process" was first identified in Fröhlich et al. (2006a), and the term was introduced by Fröhlich et al. (2006b) and is synonymous with the "neutrino-induced rp-process" in the subsequent works (Pruet et al. 2006; Wanajo 2006). This is a similar process to the classical rp-process first proposed by Wallace \& Woosley (1981). The $v p$-process is, however, essentially a new nucleosynthetic process exhibiting a number of different aspects compared with the classical rp-process. The $v p$-process starts with the seed nucleus ${ }^{56} \mathrm{Ni}$ (not ${ }^{64} \mathrm{Ge}$, the first $\beta^{+}$-waiting-point nucleus in the classical rp-process pathway), assembled from free nucleons in nuclear quasi-equilibrium (QSE) during the initial high-temperature phase $\left(T_{9}>4\right.$; where $T_{9}$ is the temperature in units of $10^{9} \mathrm{~K}$ ). The $v p$-process is therefore a primary process, which needs no pre-existing seeds. When the temperature decreases below $T_{9}=3$ (defined as the onset of a $v p$-process in this study) and QSE freezes out, the $v p$-process starts.

Neutrino capture on free protons, $p\left(\bar{v}_{\mathrm{e}}, e^{+}\right) n$, in a proton-rich neutrino-driven wind gives rise to a tiny amount of free neutrons $\left(10^{-11}\right.$ to $10^{-12}$ in mass fraction). These neutrons immediately induce the exchange reaction, $(n, p)$, and in part radiative neutron capture, $(n, \gamma)$, on the seed nucleus ${ }^{56} \mathrm{Ni}$ and subsequent heavier nuclei with decay timescales of a few milliseconds, well below the expansion timescale of the wind and well below the $\beta^{+}$-decay lifetimes of these nuclei. The nuclear flow proceeds with combination of radiative proton captures, $(p, \gamma)$, and neutron captures, the latter replacing the role of $\beta^{+}$-decays in the classical rp-process.

A large number of free protons relative to that of ${ }^{56} \mathrm{Ni}$ at $T_{9}=3$, which allows for neutron capture on the seed nuclei, are required to initiate the $v p$-process. High entropy and short expansion timescale of the ejecta make the triple- $\alpha$ process, bridging from light $(A<12)$ to heavy $(A \geqslant 12)$ nuclei, less effective and help to leave a large number of free protons at the onset of the $v p$-process. It should be noted, however, that proton-rich matter freezing out from nuclear statistical equilibrium (NSE) mainly consists of ${ }^{56} \mathrm{Ni}$ and free protons (Seitenzahl et al. 2008). This is a fundamental difference from a (moderately) neutron-rich NSE, where no free neutrons exist at freezeout. This makes the requirements for entropy and expansion timescale less crucial, compared with the case of $r$-process, allowing for the $v p$-process taking place with typical wind conditions (Fröhlich et al. 2006a; Pruet et al. 2006; Wanajo 2006).

Unlike the $r$-process, the $v p$-process is not terminated by the exhaustion of free protons, but by the temperature decrease below $T_{9}=1.5$ (defined as the end of a $v p$-process), where proton capture slows due to the Coulomb barrier. The end of $v p$-processing is thus a proton-rich freezeout. For this reason, the proton-to-seed ratio, $Y_{\mathrm{p}} / Y_{\mathrm{h}}$ (the number per nucleon for free protons divided by that for nuclei with $Z>2$ ) at $T_{9}=3$ does not necessary serve as a useful guide for the strength of the $v p$-process as the neutron-to-seed ratios are in the case of the $r$-process. Rather, the number ratio of free neutrons created by $p\left(\bar{v}_{\mathrm{e}}, e^{+}\right) n$ (for $T_{9} \leqslant 3$ ) relative to the seed nuclei (at $T_{9}=3$ ), $\Delta_{\mathrm{n}}$, can be a useful (but still crude) measure for the $v p$-process as proposed by Pruet et al. (2006). Note that each neutron capture by $(n, p)$ is immediately followed by one or two radiative proton captures, increasing the atomic masses by one or two units. Similar to Equation (2) in Pruet et al. (2006), we define

$$
\Delta_{\mathrm{n}}=\frac{Y_{\mathrm{p}} n_{\overline{\mathrm{v}}_{\mathrm{e}}}}{Y_{\mathrm{h}}},
$$

where $Y_{\mathrm{p}}$ (equal to the mass fraction of free protons, $X_{\mathrm{p}}$ ) and $Y_{\mathrm{h}}$ are the values at $T_{9}=3$. The net number of $\bar{v}_{\mathrm{e}}$ captured per free proton for $T_{9} \leqslant 3, n_{\bar{v}_{\mathrm{e}}}$, is defined as

$$
n_{\bar{\nu}_{\mathrm{e}}}=\int_{T_{9} \leqslant 3} \lambda_{\bar{v}_{\mathrm{e}}} d t,
$$

where $\lambda_{\bar{v}_{\mathrm{e}}}$ is the rate for $p\left(\bar{v}_{\mathrm{e}}, e^{+}\right) n$. The seed, a double magic nucleus ${ }^{56} \mathrm{Ni}$, remains the most abundant heavy nucleus throughout the $v p$-process. Therefore, only a fraction of ${ }^{56} \mathrm{Ni}$ is consumed for the production of heavier nuclei. For this reason, $\Delta_{n} \sim 10$ is enough for the production of nuclei with $A \sim 100-110$, as we will see in the subsequent sections.

The $v p$-process flow passes through the even-even nuclei up to $Z=N \sim 40$ and gradually deviates toward the $Z<N$ region. As the flow proceeds toward higher $Z$ nuclei, and as the temperature decreases, $(n, \gamma)$ competes with $(n, p)$. When $\Delta_{\mathrm{n}}$ is large enough, the flow eventually approaches the $\beta$-stability line and even crosses into the neutron-rich region. The latter happens when the net number of $\bar{v}_{\mathrm{e}}$ captured per free proton after the $v p$-process, defined as

$$
n_{\bar{\nu}_{\mathrm{e}}}^{\prime}=\int_{T_{9} \leqslant 1.5} \lambda_{\bar{\nu}_{\mathrm{e}}} d t,
$$

is not negligible compared to $n_{\bar{v}_{\mathrm{e}}}$. The end point of the $v p$-process is thus determined by the supernova dynamics, which enters into Equation (3) through $\lambda_{\bar{v}_{\mathrm{e}}} \propto r^{-2}$ ( $r$ is the radius from the center), rather than by the nature of nuclear physics as in case of the classical rp-process.

\section{NEUTRINO-DRIVEN WIND MODEL AND REACTION NETWORK}

The thermodynamic trajectories of neutrino-driven outflows are obtained using a semi-analytic, spherically symmetric, general relativistic model of neutrino-driven winds. This model has been developed in previous $r$-process (Wanajo et al. 2001; Wanajo 2007) and $v p$-process (Wanajo 2006) studies. Here, we describe several modifications added to the previous version.

The equation of state for ions (ideal gas) and arbitrarily degenerate, arbitrarily relativistic electrons, and positrons is taken from Timmes \& Swesty (2000). The rms-averaged energies of neutrinos are taken to be 12,14 , and $14 \mathrm{MeV}$, for electrons, anti-electrons, and the other types of neutrinos, respectively, in light of a recent self-consistently exploding model of a $9 M_{\odot}$ star (Kitaura et al. 2006; Hüdepohl et al. 2010; Müller et al. 2010). These values are consistent with other recent studies for more massive progenitors (Fischer et al. 2010), but substantially smaller than those taken in previous works (e.g., 12, 22, and $34 \mathrm{MeV}$ in Wanajo et al. 2001). The mass ejection rate $\dot{M}$ at the neutrino sphere is determined such that the outflow becomes supersonic (i.e., wind) through the sonic point. 


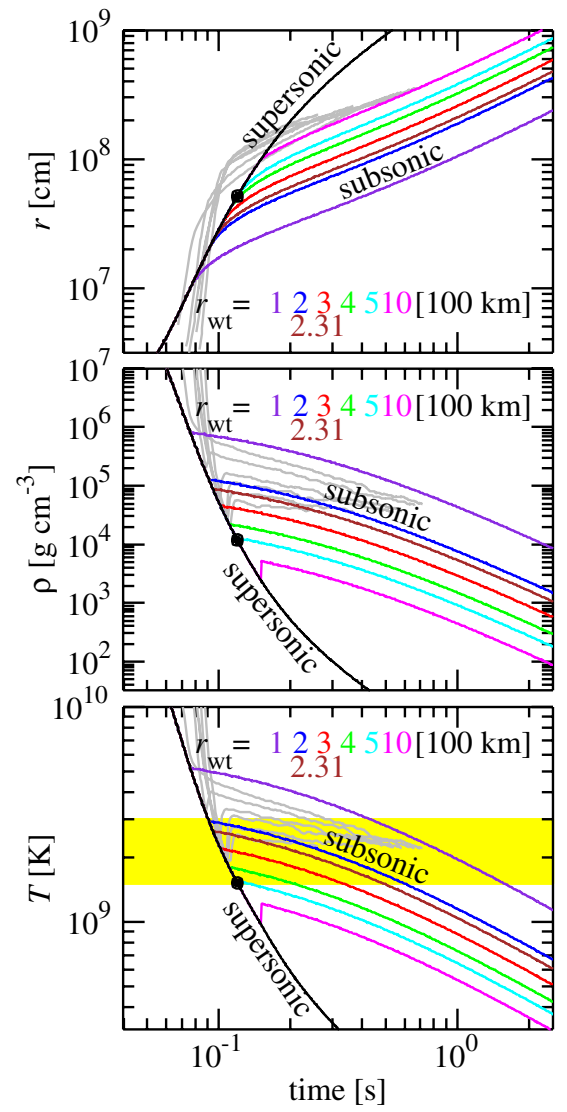

Figure 1. Radius (top), density (middle), and temperature (bottom) as a function of time (set to zero at the neutrino sphere) for $M_{\mathrm{ns}}=1.4 M_{\odot}$ and $L_{v}=1 \times 10^{52} \mathrm{erg} \mathrm{s}^{-1}$. Subsonic outflows after wind termination at $r_{\mathrm{wt}}=100,200,231,300,400,500$, and $1000 \mathrm{~km}$ are color coded. The black line shows the supersonic outflow without wind termination. In each panel, a filled circle marks the sonic point. The yellow band in the bottom panel indicates the temperature range $\left(T_{9}=1.5-3\right)$ relevant to the $v p$-process. The wind trajectories from hydrodynamical results by Buras et al. (2006, gray lines), used in Pruet et al. (2006), are compared with our models.

(A color version of this figure is available in the online journal.)

The neutron star mass $M_{\mathrm{ns}}$ is taken to be $1.4 M_{\odot}$ for our standard model. The radius of the neutrino sphere is assumed to be $R_{\nu}\left(L_{v}\right)=\left(R_{\nu 0}-R_{\nu 1}\right)\left(L_{v} / L_{\nu 0}\right)+R_{\nu 1}$ as a function of the neutrino luminosity $L_{v}$ (taken to be the same for all the flavors), where $R_{\nu 0}=30 \mathrm{~km}, R_{\nu 1}=10 \mathrm{~km}$, and $L_{\nu 0}=$ $10^{52.6}=3.98 \times 10^{52} \mathrm{erg} \mathrm{s}^{-1}$. This roughly mimics the evolution of $R_{v}$ in recent hydrodynamic simulations (e.g., Buras et al. 2006; Arcones et al. 2007). The wind solution is obtained with $L_{v}=1 \times 10^{52} \mathrm{erg} \mathrm{s}^{-1}\left(R_{v}=12.5 \mathrm{~km}\right)$ for the standard model. The time variations of radius $r$ from the center, density $\rho$, and temperature $T$ for the standard model are shown in Figure 1 (black line).

The time variations of $r, \rho$, and $T$ after the wind termination by the preceding supernova ejecta are calculated as follows. This phase is governed by the evolution of the preceding slowly outgoing ejecta, independent of the wind solution. In light of recent hydrodynamical calculations (e.g., Arcones et al. 2007), we assume the time evolution of the outgoing ejecta to be $\rho \propto t^{-2}$ and $T \propto t^{-2 / 3}$, where $t$ is the post-bounce time. With these relations, we have

$$
\rho(t)=\rho_{\mathrm{wt}}\left(\frac{t}{t_{\mathrm{wt}}}\right)^{-2}
$$

$$
\begin{gathered}
T(t)=T_{\mathrm{wt}}\left(\frac{t}{t_{\mathrm{wt}}}\right)^{-\frac{2}{3}}, \\
r(t)=r_{\mathrm{wt}}\left[1-\frac{u_{\mathrm{wt}} t_{\mathrm{wt}}}{r_{\mathrm{wt}}}+\frac{u_{\mathrm{wt}} t_{\mathrm{wt}}}{r_{\mathrm{wt}}}\left(\frac{t}{t_{\mathrm{wt}}}\right)^{3}\right]^{\frac{1}{3}}, \\
u(t)=u_{\mathrm{wt}}\left[1-\frac{u_{\mathrm{wt}} t_{\mathrm{wt}}}{r_{\mathrm{wt}}}+\frac{u_{\mathrm{wt}} t_{\mathrm{wt}}}{r_{\mathrm{wt}}}\left(\frac{t}{t_{\mathrm{wt}}}\right)^{3}\right]^{-\frac{2}{3}}\left(\frac{t}{t_{\mathrm{wt}}}\right)^{2},
\end{gathered}
$$

for $t>t_{\mathrm{wt}}$, where $t_{\mathrm{wt}}, u_{\mathrm{wt}}, r_{\mathrm{wt}}, \rho_{\mathrm{wt}}$, and $T_{\mathrm{wt}}$ are the time, velocity, radius, density, and temperature, respectively, just after the wind termination. Equation (7) represents the time variation of velocity after the wind termination. In case $r_{\mathrm{wt}}$ is larger than that at the sonic point, $r_{\mathrm{s}}$, the Rankine-Hugoniot shock-jump conditions are applied at $r_{\mathrm{wt}}$ to obtain $u_{\mathrm{wt}}, \rho_{\mathrm{wt}}$, and $T_{\mathrm{wt}}$ (see, e.g., Arcones et al. 2007; Kuroda et al. 2008). Equations (6) and (7) are obtained from Equation (4) with the steady-state condition, i.e., $r^{2} \rho u=$ constant (see Panov \& Janka 2009). Note that Equations (6) and (7) give $r(t) \propto t$ and $u(t)=$ constant for $t \gg t_{\mathrm{wt}}$. In order to obtain $t$ in Equations (4)-(7) for a given trajectory with $L_{v}$, the time evolution of $L_{v}$ at the neutrino sphere is assumed to be $\left[L_{v}(t)\right]_{r=R_{v}}=L_{v 0}\left(t / t_{0}\right)^{-1}$, where $t>t_{0}=0.2 \mathrm{~s}$ (Wanajo 2006). With this relation, the postbounce time is determined to be $t=\left(L_{v 0} / L_{v}\right) t_{0}+t_{\text {loc }}$, where $t_{\text {loc }}$ is the local time in each wind trajectory $\left(t_{\text {loc }}=0\right.$ at the neutrino sphere). The curves for various $r_{\mathrm{wt}}$ as a function of $t_{\mathrm{loc}}$ obtained from Equations (4)-(6) are shown in Figure 1.

The wind trajectories from a hydrodynamical result by Buras et al. (2006, 0.7-1.3 s after bounce, gray lines), used in Pruet et al. (2006), are compared with our models. Their wind trajectories were obtained by mapping the two-dimensional model of an exploding $15 M_{\odot}$ star to a one-dimensional grid at $\sim 0.5 \mathrm{~s}$ after bounce. In Figure 1 , the time coordinate for each trajectory is shifted to roughly match one of our models. We find that their model also exhibits a wind termination at $r \sim 500-1000 \mathrm{~km}$. The temperature and density histories are, however, close to our models with $r_{\mathrm{wt}}=100-230 \mathrm{~km}$. This is due to their higher neutrino luminosity $\left(\sim 2 \times 10^{52} \mathrm{erg} \mathrm{s}^{-1}\right)$ during the relevant core-bounce time, a factor of two higher than assumed in our models shown in Figure 1. This leads to a larger radius for a given wind temperature (see Section 4.2 and Table 1).

The nucleosynthetic abundances in the neutrino-driven outflows are calculated in a post-processing step by solving an extensive nuclear reaction network code. The network consists of 6300 species between the proton- and neutron-drip lines predicted by the recent fully microscopic mass formula (HFB-9; Goriely et al. 2005), all the way from single neutrons and protons up to the $Z=110$ isotopes. All relevant reactions, i.e., $(n, \gamma)$, $(p, \gamma),(\alpha, \gamma),(p, n),(\alpha, n),(\alpha, p)$, and their inverses are included. The experimental data, whenever available, and the theoretical predictions for light nuclei $(Z<10)$ are taken from the $\mathrm{REACLIB}^{4}$ compilation. All the other reaction rates are taken from the Hauser-Feshbach rates of BRUSLIB ${ }^{5}$ (Aikawa et al. 2005) making use of experimental masses (Audi et al. 2003)

\footnotetext{
4 http://nucastro.org/reaclib.html

5 http://www.astro.ulb.ac.be/pmwiki/Brusslib/HomePage
} 
Table 1

Results for Various Wind Models

\begin{tabular}{|c|c|c|c|c|c|c|c|c|c|c|c|c|c|c|c|c|}
\hline $\begin{array}{l}M_{\mathrm{ns}} \\
\left(M_{\odot}\right)\end{array}$ & $\begin{array}{c}\log L_{v} \\
\left(\mathrm{erg} \mathrm{s}^{-1}\right)\end{array}$ & $\begin{array}{c}r_{\mathrm{wt}} \\
(100 \mathrm{~km})\end{array}$ & $Y_{\mathrm{e}, 9^{\mathrm{a}}}$ & $\begin{array}{c}\dot{M} \\
\left(10^{-4} M_{\odot}\right)\end{array}$ & $\begin{array}{c}S \\
\left(k_{\mathrm{B}}\right)\end{array}$ & $\begin{array}{c}\tau_{1}^{\mathrm{b}} \\
(\mathrm{ms})\end{array}$ & $\begin{array}{c}\tau_{2}{ }^{\mathrm{c}} \\
(\mathrm{ms})\end{array}$ & $T_{\mathrm{wt}, 9}{ }^{\mathrm{d}}$ & $Y_{\mathrm{e}, 3}{ }^{\mathrm{e}}$ & $Y_{\mathrm{p}} / Y_{\mathrm{h}}^{\mathrm{f}}$ & $n_{\bar{v}_{\mathrm{e}}}$ & $\Delta_{n}$ & $\log f_{\max }{ }^{\mathrm{g}}$ & $\operatorname{nuc}\left(f_{\max }\right)^{\mathrm{h}}$ & $\operatorname{nuc}\left(A_{\max }\right)^{\mathrm{i}}$ & Fig. \\
\hline$\overline{1.4}$ & 52.0 & 3.00 & 0.600 & 2.70 & 57.0 & 17.5 & 245 & 2.19 & 0.550 & 124 & 0.0834 & 10.3 & 7.16 & ${ }^{96} \mathrm{Ru}$ & ${ }^{106} \mathrm{Cd}$ & $\overline{\text { All }}$ \\
\hline 1.4 & 52.0 & 1.00 & 0.600 & 2.70 & 57.0 & 359 & 1160 & 5.19 & 0.509 & 1.78 & 0.135 & 0.240 & 4.44 & ${ }^{64} \mathrm{Zn}$ & ${ }^{74} \mathrm{Se}$ & 2 \\
\hline 1.4 & 52.0 & 2.00 & 0.600 & 2.70 & 57.0 & 17.5 & 516 & 2.95 & 0.550 & 124 & 0.138 & 17.1 & 6.27 & ${ }^{78} \mathrm{Kr}$ & ${ }^{84} \mathrm{Sr}$ & 2 \\
\hline 1.4 & 52.0 & 2.31 & 0.600 & 2.70 & 57.0 & 17.5 & 403 & 2.65 & 0.550 & 124 & 0.114 & 14.1 & 7.67 & ${ }^{106} \mathrm{Cd}$ & ${ }^{108} \mathrm{Cd}$ & 2 \\
\hline 1.4 & 52.0 & 3.00 & 0.600 & 2.70 & 57.0 & 17.5 & 245 & 2.19 & 0.550 & 124 & 0.0834 & 10.3 & 7.16 & ${ }^{96} \mathrm{Ru}$ & ${ }^{106} \mathrm{Cd}$ & 2 \\
\hline 1.4 & 52.0 & 4.00 & 0.600 & 2.70 & 57.0 & 17.5 & 117 & 1.80 & 0.550 & 124 & 0.0628 & 7.79 & 6.86 & ${ }^{84} \mathrm{Sr}$ & ${ }^{102} \mathrm{Pd}$ & 2 \\
\hline 1.4 & 52.0 & 5.00 & 0.600 & 2.70 & 57.0 & 17.5 & 44.0 & 1.55 & 0.550 & 124 & 0.0529 & 6.56 & 6.69 & ${ }^{84} \mathrm{Sr}$ & ${ }^{84} \mathrm{Sr}$ & 2 \\
\hline 1.4 & 52.0 & 10.0 & 0.600 & 2.70 & 57.0 & 17.5 & 30.0 & 1.21 & 0.550 & 124 & 0.0431 & 5.34 & 6.13 & ${ }^{78} \mathrm{Kr}$ & ${ }^{84} \mathrm{Sr}$ & 2 \\
\hline 1.4 & 52.0 & $\infty$ & 0.600 & 2.70 & 57.0 & 17.5 & 30.0 & $\cdots$ & 0.550 & 124 & 0.0323 & 4.01 & 5.79 & ${ }^{78} \mathrm{Kr}$ & ${ }^{84} \mathrm{Sr}$ & 2 \\
\hline 1.4 & 52.4 & 8.01 & 0.600 & 31.3 & 33.7 & 22.8 & 261 & 2.19 & 0.558 & 42.7 & 0.0611 & 2.61 & 6.00 & ${ }^{78} \mathrm{Kr}$ & ${ }^{84} \mathrm{Sr}$ & 3 \\
\hline 1.4 & 52.2 & 4.29 & 0.600 & 8.66 & 44.7 & 18.9 & 236 & 2.19 & 0.554 & 78.3 & 0.0720 & 5.64 & 6.83 & ${ }^{84} \mathrm{Sr}$ & ${ }^{96} \mathrm{Ru}$ & 3 \\
\hline 1.4 & 52.0 & 3.00 & 0.600 & 2.70 & 57.0 & 17.5 & 245 & 2.19 & 0.550 & 124 & 0.0834 & 10.3 & 7.16 & ${ }^{96} \mathrm{Ru}$ & ${ }^{106} \mathrm{Cd}$ & 3 \\
\hline 1.4 & 51.8 & 2.22 & 0.600 & 0.921 & 70.1 & 17.8 & 262 & 2.19 & 0.545 & 166 & 0.0945 & 15.7 & 7.78 & ${ }^{102} \mathrm{Pd}$ & ${ }^{108} \mathrm{Cd}$ & 3 \\
\hline 1.4 & 51.6 & 1.71 & 0.600 & 0.339 & 83.3 & 19.9 & 301 & 2.19 & 0.540 & 185 & 0.107 & 19.8 & 7.99 & ${ }^{106} \mathrm{Cd}$ & ${ }^{108} \mathrm{Cd}$ & 3 \\
\hline 1.4 & 51.4 & 1.37 & 0.600 & 0.131 & 96.3 & 24.4 & 371 & 2.19 & 0.535 & 174 & 0.121 & 21.1 & 8.07 & ${ }^{106} \mathrm{Cd}$ & ${ }^{108} \mathrm{Cd}$ & 3 \\
\hline 1.2 & 52.0 & 3.27 & 0.600 & 3.96 & 46.8 & 18.4 & 241 & 2.19 & 0.553 & 84.4 & 0.0746 & 6.30 & 6.96 & ${ }^{84} \mathrm{Sr}$ & ${ }^{102} \mathrm{Pd}$ & 4 \\
\hline 1.4 & 52.0 & 3.00 & 0.600 & 2.70 & 57.0 & 17.5 & 245 & 2.19 & 0.550 & 124 & 0.0834 & 10.3 & 7.16 & ${ }^{96} \mathrm{Ru}$ & ${ }^{106} \mathrm{Cd}$ & 4 \\
\hline 1.6 & 52.0 & 2.80 & 0.600 & 1.94 & 68.1 & 16.4 & 244 & 2.19 & 0.547 & 178 & 0.0908 & 16.2 & 7.69 & ${ }^{102} \mathrm{Pd}$ & ${ }^{108} \mathrm{Cd}$ & 4 \\
\hline 1.8 & 52.0 & 2.62 & 0.600 & 1.46 & 80.0 & 15.4 & 245 & 2.19 & 0.545 & 243 & 0.0980 & 23.8 & 7.91 & ${ }^{106} \mathrm{Cd}$ & ${ }^{108} \mathrm{Cd}$ & 4 \\
\hline 2.0 & 52.0 & 2.46 & 0.600 & 1.13 & 93.0 & 14.4 & 247 & 2.19 & 0.543 & 335 & 0.104 & 34.8 & 8.12 & ${ }^{108} \mathrm{Cd}$ & ${ }^{108} \mathrm{Cd}$ & 4 \\
\hline 1.4 & 52.0 & 3.00 & 0.550 & 2.70 & 57.0 & 17.5 & 245 & 2.19 & 0.523 & 42.9 & 0.0834 & 3.58 & 6.25 & ${ }^{78} \mathrm{Kr}$ & ${ }^{84} \mathrm{Sr}$ & 5 \\
\hline 1.4 & 52.0 & 3.00 & 0.600 & 2.70 & 57.0 & 17.5 & 245 & 2.19 & 0.550 & 124 & 0.0834 & 10.3 & 7.16 & ${ }^{96} \mathrm{Ru}$ & ${ }^{106} \mathrm{Cd}$ & 5 \\
\hline 1.4 & 52.0 & 3.00 & 0.650 & 2.70 & 57.0 & 17.5 & 245 & 2.19 & 0.576 & 245 & 0.0834 & 20.4 & 8.14 & ${ }^{106} \mathrm{Cd}$ & ${ }^{108} \mathrm{Cd}$ & 5 \\
\hline 1.4 & 52.0 & 3.00 & 0.700 & 2.70 & 57.0 & 17.5 & 245 & 2.19 & 0.603 & 428 & 0.0834 & 35.7 & 8.34 & ${ }^{108} \mathrm{Cd}$ & ${ }^{120} \mathrm{Te}$ & 5 \\
\hline 1.4 & 52.0 & 3.00 & 0.750 & 2.70 & 57.0 & 17.5 & 245 & 2.19 & 0.629 & 703 & 0.0834 & 58.6 & 8.54 & ${ }^{138} \mathrm{La}$ & ${ }^{138} \mathrm{La}$ & 5 \\
\hline 1.4 & 52.0 & 3.00 & 0.800 & 2.70 & 57.0 & 17.5 & 245 & 2.19 & 0.655 & 1130 & 0.0834 & 94.2 & 8.37 & ${ }^{138} \mathrm{La}$ & ${ }^{152} \mathrm{Gd}$ & 5 \\
\hline
\end{tabular}

Notes.

${ }^{\text {a }} Y_{\mathrm{e}}$ at $T_{9}=9$.

b Time elapsed from $T_{9}=6$ to $T_{9}=3$.

c Time elapsed from $T_{9}=3$ to $T_{9}=1.5$.

d Temperature (in units of $10^{9} \mathrm{~K}$ ) just after the wind termination.

e $Y_{\mathrm{e}}$ at $T_{9}=3$.

${ }^{\mathrm{f}}$ Proton-to-seed ratio at $T_{9}=3$.

g Maximum production factor.

h Nuclide at $f=f_{\text {max }}$.

${ }^{\mathrm{i}}$ Nuclide at the largest $A$ with $f>f_{\max } / 10$.

whenever available or the HFB-9 mass predictions (Goriely et al. 2005) otherwise. The photodisintegration rates are deduced from the reverse rates applying the reciprocity theorem with the nuclear masses considered.

The $\beta$-decay rates are taken from the gross theory predictions (GT2; Tachibana et al. 1990) obtained with the HFB-9 predictions (T. Tachibana 2005, private communication). Electroncapture reactions on free nucleons and on heavy nuclei (Fuller et al. 1982; Langanke \& Martinez-Pinedo 2001) as well as rates for neutrino capture on free nucleons and ${ }^{4} \mathrm{He}$ and for neutrino spallation of free nucleons from ${ }^{4} \mathrm{He}$ (Woosley et al. 1990; McLaughlin et al. 1996) are also included. Neutrino-induced reactions of heavy nuclei are not taken into account in this study, which are expected to make only minor effects in this study.

Each nucleosynthesis calculation is initiated when the temperature decreases to $T_{9}=9$, at which only free nucleons exist. The initial compositions are then given by the electron fraction $Y_{\mathrm{e}, 9}$ (proton-to-baryon ratio) at $T_{9}=9$, such as $Y_{\mathrm{e}, 9}$ and $1-Y_{\mathrm{e}, 9}$ for the mass fractions of free protons and neutrons, respectively.

\section{UNCERTAINTIES IN SUPERNOVA DYNAMICS}

In the following subsections, we examine how the nucleosynthesis of the $v p$-process is influenced by varying the wind-termination radius $r_{\mathrm{wt}}$ (or temperature; Section 4.1), $L_{v}$ (Section 4.2), $M_{\mathrm{ns}}$ (Section 4.3), and $Y_{\mathrm{e}, 9}$ (Section 4.4) from their fiducial values $300 \mathrm{~km}$ ( or $\left.2.19 \times 10^{9} \mathrm{~K}\right), 1 \times 10^{52} \mathrm{erg} \mathrm{s}^{-1}$, $1.4 M_{\odot}$, and 0.600 , respectively, of our standard model (first line in Table 1). These values are taken as those expected in the early wind phase of core-collapse supernovae. All the explored models and their major outcomes are summarized in Table 1 (the first four columns represent the input parameters).

\subsection{Wind-termination Radius}

Recent hydrodynamic studies of core-collapse supernovae have shown that the neutrino-driven outflows develop to be supersonic, which abruptly decelerate by the reverse shock from the outer layers (e.g., Janka \& Müller 1995, 1996; Burrows et al. 1995; Buras et al. 2006). Arcones et al. (2007) have explored the effects of the reverse shock on the properties of neutrino-driven winds by one-dimensional, long-term hydrodynamic simulations of core-collapse supernovae. Their result shows that, in all of their models (10-25 $M_{\odot}$ progenitors), the outflows become supersonic and form the termination shock when colliding with the slower preceding supernova ejecta. This condition continues until the end of their computations ( $10 \mathrm{~s}$ after core bounce) in all of their "standard" models with reasonable parameter choices. 
A recent self-consistently exploding model of a $9 M_{\odot}$ star also shows qualitatively the same result (Hüdepohl et al. 2010).

In this subsection, we explore the effect of the wind termination on the $v p$-process. The termination point is located at $r_{\mathrm{wt}}=100,200,231,300$ (standard model), 400, 500, and $1000 \mathrm{~km}$ on the transonic wind trajectory (black line) shown in Figure 1 (top panel). The other parameters $L_{v}, M_{\mathrm{ns}}$, and $Y_{\mathrm{e}, 9}$ are kept to be the fiducial values (Table 1 , second to ninth lines). In Figure 1 (middle and bottom panels), we find shock jumps of density and temperature by wind termination only for the $r_{\mathrm{wt}}=1000 \mathrm{~km}$ case, since the termination points are placed below the sonic radius $\left(r_{\mathrm{s}}=515 \mathrm{~km}\right.$; Figure 1, top panel) for the other cases. ${ }^{6}$

The result of nucleosynthesis calculations is shown in Figure 2. The top panel shows the mass fractions, $X_{A}$, of nuclei as a function of atomic mass number, $A$. We find that the case with $r_{\mathrm{wt}}=231 \mathrm{~km}$ has the maximum efficiency of producing nuclei with $A=100-110$ (including our calculations not shown here). The middle and bottom panels show, respectively, the mass fractions relative to the standard model $\left(=X_{A} / X_{A \text {,standard }}\right)$ and to their solar values (Lodders 2003), i.e., the production factor $f\left(=X_{i} / X_{i, \odot}\right.$ for $i$ th isotope), as a function of $A$. We find a noticeable effect of wind termination on the $v p$-process; the production of $p$-nuclei between $A=90$ and 110 is outstanding for the cases with $r_{\mathrm{wt}}=231$ and $300 \mathrm{~km}$ (standard model).

It should be noted that the asymptotic entropy $S(=57.0$ per nucleon in units of the Boltzmann constant $k_{\mathrm{B}}$; Table 1) is the same for all the cases here (except for $r_{\mathrm{wt}}=1000 \mathrm{~km}$ owing to the termination-shock heating). These different outcomes can be explained by the different values of $\Delta_{\mathrm{n}}(=0.24-17,13$ th column in Table 1), defined by Equation (1), owing to the different expansion timescales after wind termination. As indicated by the yellow band in Figure 1 (bottom panel), we find substantial differences in the temperature histories before or during the $v p$-process phase (defined as $T_{9}=1.5-3$ ).

We define two expansion timescales $\tau_{1}$ and $\tau_{2}$ (seventh and eighth columns in Table 1); the former is the time elapsed from $T_{9}=6$ to $T_{9}=3$ and the latter from $T_{9}=3$ to $T_{9}=1.5$. These represent the durations of the seed production and of the $v p$-process, respectively. As can be seen in Figure 1 (bottom panel), $\tau_{1}(=17.5 \mathrm{~ms})$ and thus the proton-to-seed ratio $Y_{\mathrm{p}} / Y_{\mathrm{h}}$ (=124) at $T_{9}=3$ are the same except for the case with $r_{\mathrm{wt}}=100 \mathrm{~km}$. Nevertheless, the different values of $\tau_{2}$ and thus $\Delta_{\mathrm{n}}$ (see Equation (1)) lead to the different efficiencies of the $v p$-process. We find that $\Delta_{\mathrm{n}} \sim 10$ is needed for an efficient production of $p$-nuclei with $A \sim 100$. This requires the wind termination at $T_{\mathrm{wt}, 9} \sim 2-3$ (in units of $10^{9} \mathrm{~K}$ ) to obtain $n_{\bar{v}_{\mathrm{e}}} \sim 0.1$ (Equation (2)). For the standard model $\left(r_{\mathrm{wt}}=300 \mathrm{~km}\right.$ and $\left.T_{\mathrm{wt}, 9}=2.19\right)$, the maximum production factor $\left(f_{\max }\right.$ in Table 1$)$ is obtained at ${ }^{96} \mathrm{Ru}\left(\operatorname{nuc}\left(f_{\max }\right)\right.$ in Table 1$)$, a daughter nucleus of ${ }^{96} \mathrm{Pd}(N=50)$ on the $v p$-process pathway. We have the optimal production $\left(\log f_{\max }=7.67\right.$ at $\left.{ }^{106} \mathrm{Cd}\right)$ with $T_{\mathrm{wt}, 9}=2.65$ when the termination point is set to $r_{\mathrm{wt}}=231 \mathrm{~km}$.

In Table 1, the nuclide with the largest mass number $A_{\max }$ with $f>f_{\max } / 10$ is also shown (e.g., ${ }^{106} \mathrm{Cd}$ for the standard model; $\operatorname{nuc}\left(A_{\max }\right)$ in Table 1$)$, which is taken to be the largest $A$ of the

\footnotetext{
6 The outflows with $r_{\mathrm{wt}}<r_{\mathrm{S}}$ are subsonic all the way. This happens in the early wind phase when the slowly outgoing ejecta is still close to the core (Arcones et al. 2007). In this case, however, the mass ejection rate from the core is expected to be close to that of the transonic solution (with the maximum $\dot{M}$ ). Thus, the time variations of $r, \rho$, and $T$ may not be substantially different from those of the transonic case for $r<r_{\mathrm{wt}}$ (see, e.g., Otsuki et al. 2000). We take, therefore, the transonic solution for all the cases, rather than the subsonic solution by introducing an additional free parameter $\dot{M}$.
}
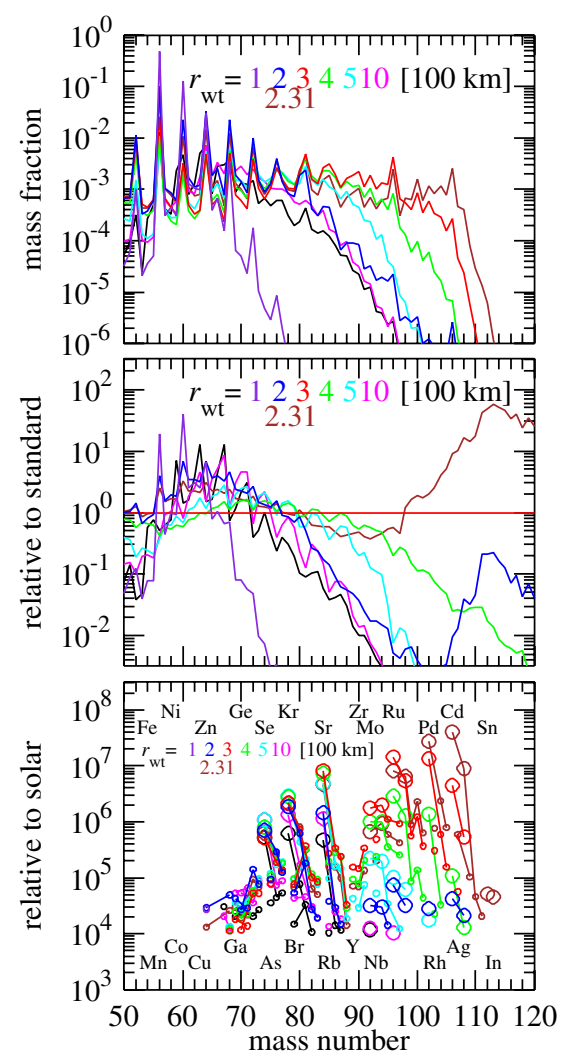

Figure 2. Comparison of the nucleosynthetic results for various windtermination radii $r_{\mathrm{wt}}$. The mass fractions (top) and their ratios relative to those for the standard model (middle) are shown as a function of atomic mass number. The bottom panel shows the abundances of isotopes (connected by a line for a given element) relative to their solar values, where those lower than $10^{4}$ are omitted. The color coding corresponds to different values of $r_{\mathrm{wt}}$ as indicated in each panel (red is the standard model). The result for the outflow without wind termination is shown in black. In the bottom panel, the names of elements are specified in the upper (even $Z$ ) and lower (odd $Z$ ) sides at their lightest mass numbers.

(A color version of this figure is available in the online journal.)

$p$-nuclei synthesized by the $v p$-process. Given that our standard model represents a typical supernova condition, this implies that the $v p$-process can be the source of the solar $p$-abundances up to $A \sim 110$ (see Section 6 for more detail). However, this favorable condition is not robust against a variation of $r_{\mathrm{wt}}$ (and thus $\left.T_{\mathrm{wt}}\right)$; the outflows with $r_{\mathrm{wt}}=200 \mathrm{~km}\left(T_{\mathrm{wt}, 9}=2.95\right)^{7}$ and $r_{\mathrm{wt}} \geqslant 500 \mathrm{~km}\left(T_{\mathrm{wt}, 9}<1.55\right)$ end up with $A_{\max }=84\left({ }^{84} \mathrm{Sr}\right.$; Table 1). Note that the outflow with $r_{\mathrm{wt}}=1000 \mathrm{~km}$ leads to a similar result as that without wind termination (black line in Figure $2 ; r_{\mathrm{wt}}=\infty$ in Table 1 ). This indicates that the role of wind termination is unimportant for $T_{\mathrm{wt}, 9}<1.5$.

We find no substantial $v p$-processing for the outflow with $r_{\mathrm{wt}}=100 \mathrm{~km}$ (Figure 2). This is due to the substantially smaller $Y_{\mathrm{e}}$ at the beginning of the $v p$-process $\left(T_{9}=3\right), Y_{\mathrm{e}, 3}=0.509$ (only slightly proton-rich), than those for the other cases $(0.550$; Table 1). As a result, $Y_{\mathrm{p}} / Y_{\mathrm{h}}$ at $T_{9}=3$ is only 1.78 , resulting in a small $\Delta_{\mathrm{n}}(=0.24)$ in spite of the largest $n_{\bar{\nu}_{\mathrm{e}}}$ among the various $r_{\mathrm{wt}}$ models presented here. It should be noted that $Y_{\mathrm{e}, 3}$ is always lower than $Y_{\mathrm{e}, 9}(=0.600$ in the present cases $)$. This is due to a couple of neutrino effects. One is that the

\footnotetext{
7 Despite the largest $\Delta_{\mathrm{n}}(=17.1)$ among the various $r_{\mathrm{wt}}$ models, the $r_{\mathrm{wt}}=200 \mathrm{~km}$ model ends up with inefficient $v p$-processing. This is due to $\Delta_{\mathrm{n}}$ defined for $T_{9} \leqslant 3$ (Equation (1)), while the maximal efficiency of $v p$-processing is obtained with $T_{\mathrm{wt}}=2.65$ in this case.
} 
asymptotic equilibrium value of $Y_{\mathrm{e}}$ in the non-degenerate matter consisting of free nucleons, which is subject to neutrino capture, is $Y_{\mathrm{e}, \mathrm{a}} \approx 0.56$ (see, e.g., Qian \& Woosley 1996) with the neutrino luminosities and energies taken in this study. Hence, the value starts relaxing from $Y_{\mathrm{e}, 9}$ toward $Y_{\mathrm{e}, \mathrm{a}}$ as soon as the calculation initiates. The other effect is due to the continuous $\alpha$ particle formation $\left(T_{9}<7\right)$ from inter-converting free protons and free neutrons that is subject to neutrino capture, which drives $Y_{\mathrm{e}}$ toward 0.5 (" $\alpha$-effect"; Meyer et al. 1998b). In the $r_{\mathrm{wt}}=100 \mathrm{~km}$ case, the wind termination takes place at high temperature $\left(T_{\mathrm{wt}, 9}=5.19\right)$ and thus the long $\tau_{1}(=359 \mathrm{~ms})$ leads to the low $Y_{\mathrm{e}, 3}$ owing to the neutrino effects.

In summary, our exploration here elucidates a crucial role of wind termination on the $v p$-process. On one hand, a fast expansion above the temperature $T_{9} \sim 3$ (more precisely, $T_{9}=2.65$ in the considered conditions) is favored to obtain a high proton-to-seed ratio at the onset of the $v p$-process. On the other hand, a slow expansion below this temperature, owing to wind termination, is needed to obtain $\Delta_{\mathrm{n}} \sim 10$ for efficient $v p$-processing.

We presume that the reason for somewhat different outcomes in previous studies of the $v p$-process described in Section 1 is largely due to their different behaviors of wind termination. The temperature histories of trajectories taken by Pruet et al. (2006, an exploding $15 M_{\odot}$ star), similar to our models with $r_{\mathrm{wt}}=100-230\left(T_{\mathrm{wt}, 9}=2.7-5.2\right)$, lead to the production of $p$-nuclei up to $A \sim 100$. The reason of weak $v p$-processing in Fröhlich et al. (2006a; a $20 M_{\odot}$ explosion) may be rather due to the moderate proton richness (up to $Y_{\mathrm{e}} \sim 0.54$ ) in their simulations (see Section 4.4 and Figure 6). In contrast, negligible production of $p$-nuclei in the electron-capture supernova of a $9 M_{\odot}$ star (Wanajo et al. 2009, also S. Wanajo et al. 2011, in preparation) is due to the absence of a wind-termination shock within the relevant temperature range $\left(T_{9}=1.5-3\right)$ owing to the steep density gradient of the oxygen-neon-magnesium core progenitors surrounded by a diluted outer $\mathrm{H} / \mathrm{He}$ envelope.

\subsection{Neutrino Luminosity}

The neutrino luminosity $L_{v}$ decreases with time from its initial value of a few $10^{52} \mathrm{erg} \mathrm{s}^{-1}$ to $\sim 10^{51} \mathrm{erg} \mathrm{s}^{-1}$ during the first $10 \mathrm{~s}$ (Fischer et al. 2010; Hüdepohl et al. 2010). In this subsection, we examine the effect of $L_{v}$ on the $v p$-process, by varying its value from $10^{52.4}=2.51 \times 10^{52} \mathrm{erg} \mathrm{s}^{-1}$ to 10 times smaller than that with an interval of 0.2 dex (from 10th to 15th lines in Table 1 and Figure 3). $M_{\mathrm{ns}}$ and $Y_{\mathrm{e}, 9}$ are taken to be the fiducial values of $1.4 M_{\odot}$ and 0.600 , respectively. In Section 4.1, we found that the temperature at the wind termination, $T_{\mathrm{wt}}$, plays a crucial role for the $v p$-process. Hence, we adjust $r_{\mathrm{wt}}$ (Table 1) such that the fiducial value of $T_{\mathrm{wt}, 9}=2.19$ is obtained for each $L_{v}$.

The results of nucleosynthesis calculations are shown in Figure 3 and Table 1. We clearly see the increasing efficiency of $v p$-processing with a decrease of $L_{v}$. This is due to the larger entropy for a smaller $L_{v}$ (Table 1), while the expansion timescales $\tau_{1}$ (prior to the $v p$-process) are similar. ${ }^{8}$ This leads to a higher $Y_{\mathrm{p}} / Y_{\mathrm{h}}$ at the onset of the $v p$-process for a lower $L_{v}$. In addition, the somewhat larger timescale $\tau_{2}$ for a smaller

\footnotetext{
8 When the radius of the neutrino sphere $R_{v}$ is fixed to a constant value, the expansion timescale increases with decreasing $L_{v}$ (see, e.g., Otsuki et al. 2000; Wanajo et al. 2001). In this study, however, $R_{v}$ is assumed to decrease with decreasing $L_{v}$ (Section 2), which is more realistic. As a result, the difference of $\tau_{1}$ in the range of $L_{v}$ explored here is moderate.
}
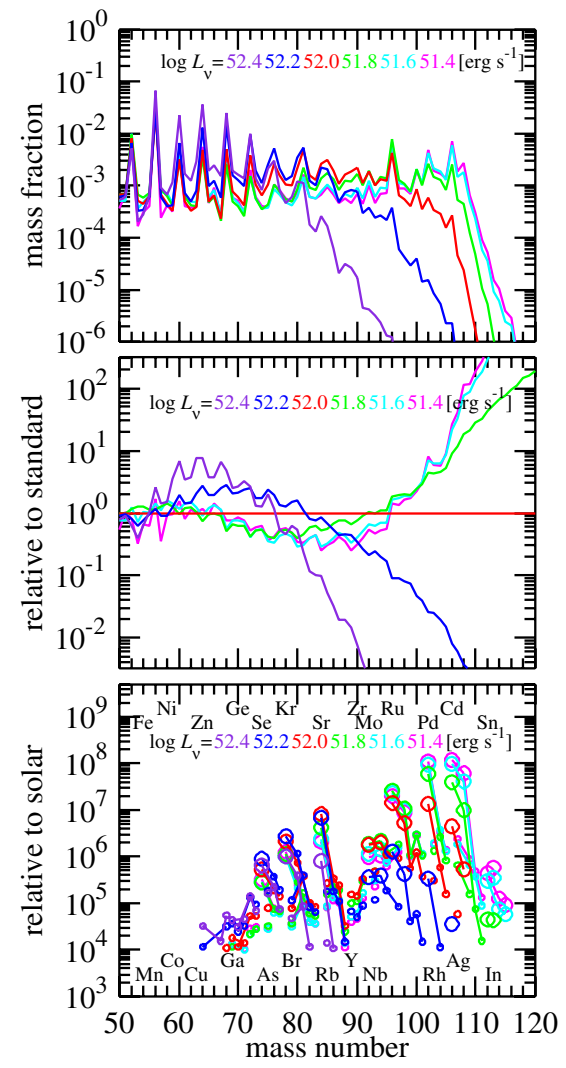

Figure 3. Same as Figure 2, but for various neutrino luminosities $\left(L_{v}\right)$. (A color version of this figure is available in the online journal.)

$L_{\nu}$ increases $n_{\bar{\nu}_{\mathrm{e}}}$ (12th column in Table 1). For these reasons, a smaller $L_{v}$ model achieves larger $\Delta_{\mathrm{n}}$, leading to a more efficient $v p$-process.

It should be noted that in our explored cases, $r_{\mathrm{wt}}$ decreases with decreasing $L_{v}$ (Table 1 ) in order to obtain the fiducial value of $T_{\mathrm{wt}, 9}=2.19$ (to figure out solely the effect of $L_{v}$ ). However, if $r_{\mathrm{wt}}$ increases with time and thus $T_{\mathrm{wt}}$ decreases with decreasing $L_{v}$, as in many explosion models, only the early stage of the neutrino-driven wind with $L_{v} \sim 10^{52} \mathrm{erg} \mathrm{s}^{-1}$ may be relevant to the high $T_{\mathrm{wt}, 9}=1.5-3$ (see, e.g., Arcones et al. 2007) that is needed for efficient $v p$-processing (Section 4.1).

\subsection{Neutron Star Mass}

The mass of the proto-neutron star $M_{\mathrm{ns}}$ can be somewhat different from its canonical value of $1.4 M_{\odot}$, depending on its progenitor mass. In this subsection, we examine the nucleosynthesis calculations with $M_{\mathrm{ns}}=1.2,1.4,1.6,1.8$, and $2.0 M_{\odot}$, while $L_{v}$ and $Y_{\mathrm{e}, 9}$ are kept to be their fiducial values of $10^{52} \mathrm{erg} \mathrm{s}^{-1}$ and 0.600 . For each case, the fiducial value of $T_{\mathrm{wt}, 9}=2.19$ is obtained by adjusting $r_{\mathrm{wt}}$ (from 16th to 20th lines in Table 1) as in Section 4.2.

We find a clear correlation between an increase of $M_{\mathrm{ns}}$ and an increasing efficiency of $v p$-processing in Figure 4 and Table 1. This is due to a larger $S$ and a smaller $\tau_{1}$ for a larger $M_{\mathrm{ns}}$ (e.g., Otsuki et al. 2000; Wanajo et al. 2001), both of which help to increase $Y_{\mathrm{p}} / Y_{\mathrm{h}}$ and thus $\Delta_{\mathrm{n}}$. This means that a more massive progenitor (up to $\sim 30 M_{\odot}$, which forms a neutron star) is favored for the $v p$-process, given that all the other parameters are the same. In reality, however, other factors, such as the evolutions of $L_{v}, r_{\mathrm{wt}}$, and $Y_{\mathrm{e}}$ should be dependent on the progenitor mass (e.g., Arcones et al. 2007), which prevents us 

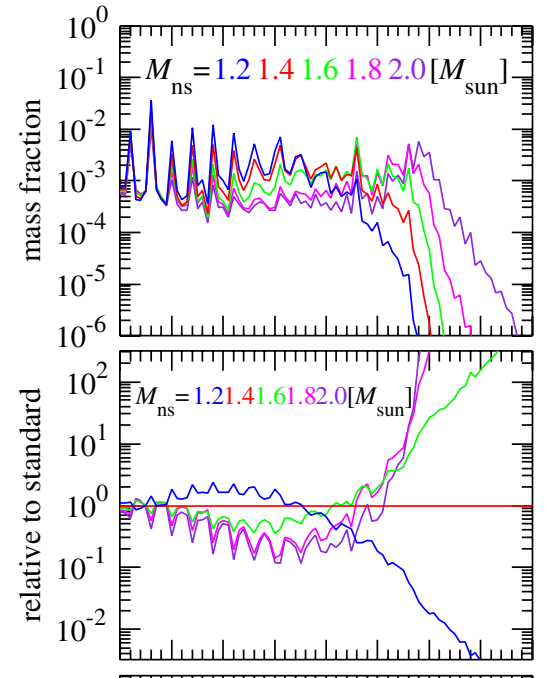

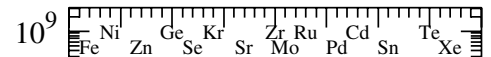

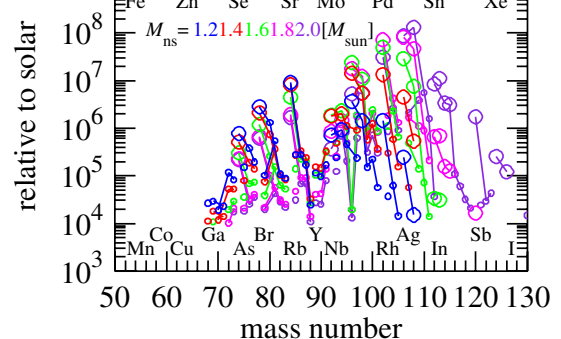

Figure 4. Same as Figure 2, but for various neutron star masses $\left(M_{\mathrm{ns}}\right)$.

(A color version of this figure is available in the online journal.)

from drawing any firm conclusions. It should be emphasized, however, that the outflow with a typical mass of $M_{\mathrm{ns}}=1.4 M_{\odot}$ can already provide physical conditions sufficient for producing the $p$-nuclei up to $A \sim 110$.

\subsection{Electron Fraction}

The electron fraction $Y_{\mathrm{e}}$ is obviously one of the most important ingredients in the $v p$-process as it controls the proton richness in the ejecta. Recent hydrodynamical studies with elaborate neutrino transport indicate that $Y_{\mathrm{e}}$ exceeds 0.5 and increases up to $\sim 0.6$ during the neutrino-driven wind phase (Fischer et al. 2010; Hüdepohl et al. 2010). It should be noted that $Y_{\mathrm{e}}$ substantially decreases from its initial value owing to the neutrino effects (Section 4.1). In our standard model, the value decreases from $Y_{\mathrm{e}, 9}=0.600$ (at $T_{9}=9$ ) to $Y_{\mathrm{e}, 3}=0.550$ at the onset of the $v p$-process $\left(T_{9}=3\right)$. However, these neutrino effects would be highly dependent on the neutrino luminosities and energies of electron and anti-electron neutrinos assumed in this study. In this subsection, therefore, we take the value at the onset of the $v p$-process, $Y_{\mathrm{e}, 3}$, as a reference, rather than the initial value $Y_{\mathrm{e}, 9}$.

Figure 5 and Table 1 (the last six lines) show the nucleosynthetic results for $Y_{\mathrm{e}, 3}=0.523,0.550,0.576,0.603,0.629$, and 0.655 (see Table 1 for their initial values $Y_{\mathrm{e}, 9}$ ). The other parameters $M_{\mathrm{ns}}, L_{v}$, and $r_{\mathrm{wt}}$ (and thus $T_{\mathrm{wt}}$ ) are kept to be their fiducial values (first line in Table 1). We find a great impact of the $Y_{\mathrm{e}}$ variation; an increase of only $\Delta Y_{\mathrm{e}, 3} \sim 0.03$ leads to a 10 unit increase of $A_{\max }$, while $f_{\max }$ is similar for $Y_{\mathrm{e}, 3}>0.550$. This is due to the larger $Y_{\mathrm{p}} / Y_{\mathrm{h}}\left(\right.$ at $\left.T_{9}=3\right)$ for a larger $Y_{\mathrm{e}, 3}$, leading the larger $\Delta_{n}$ despite the same $n_{\bar{\nu}_{\mathrm{e}}}$ (Table 1).

In order to elucidate the effect of $Y_{\mathrm{e}}$ in more detail, the production factor $f$ for each $p$-nucleus is drawn in Figure 6
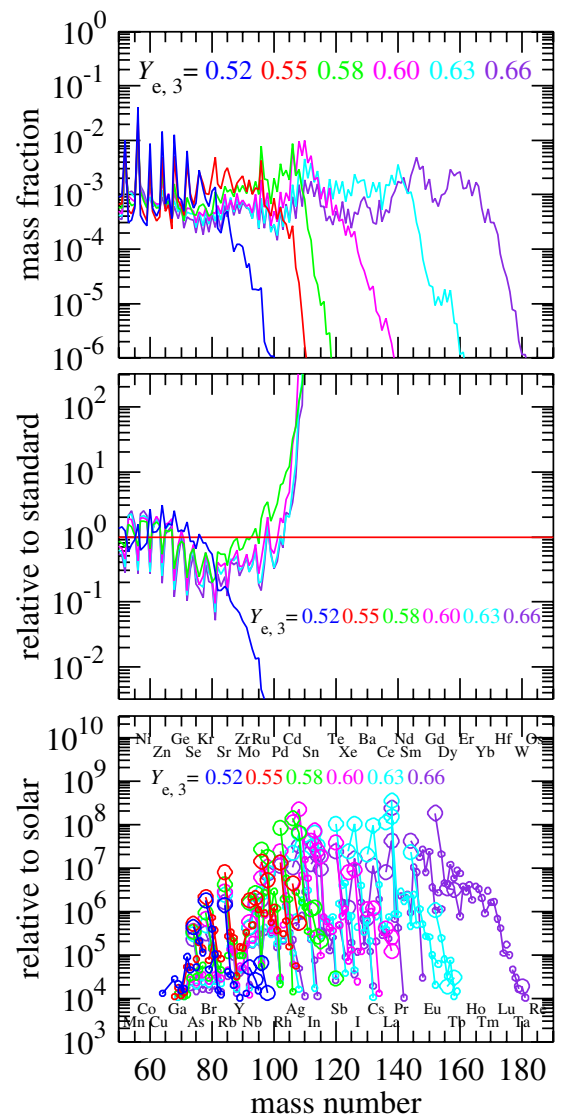

Figure 5. Same as Figure 2, but for various electron fractions $\left(Y_{\mathrm{e}, 3}\right)$. (A color version of this figure is available in the online journal.)

as a function of $Y_{\mathrm{e}, 3}$, where $M_{\mathrm{ns}}, L_{v}$, and $r_{\mathrm{wt}}$ are kept to be their fiducial values. Each element is color coded with the solid, dashed, and long-dashed lines for the lightest, second-lightest, and third-lightest ( ${ }^{115} \mathrm{Sn}$ is only the case) isotopes, respectively (see the first column of Table 4 for the list of $p$-nuclei). We find in the top panel of Figure 6 that the $p$-nuclei up to $A=$ $108\left({ }^{108} \mathrm{Cd}\right)$ take the maximum production factors between $Y_{\mathrm{e}, 3}=0.53$ and 0.60. Given the maximum $Y_{\mathrm{e}, 3}$ to be $\sim 0.6$ according to some recent hydrodynamic results (e.g., Fischer et al. 2010; Hüdepohl et al. 2010), this implies that the maximum mass number of the $p$-nuclei produced by the $v p$-process is $A \sim 110$.

In principle, the heavier $p$-nuclei can be synthesized if the matter is more proton-rich than $Y_{\mathrm{e}, 3}=0.6$. The middle panel of Figure 6 shows that the production factors of the $p$-nuclei from $A=113\left({ }^{113} \mathrm{In}\right)$ up to $A=138\left({ }^{138} \mathrm{Ce}\right)$ are maximal between $Y_{\mathrm{e}, 3}=0.61$ and 0.63 . Furthermore, ${ }^{144} \mathrm{Sm}$ and ${ }^{152} \mathrm{Gd}$ reach the maximum production factors at $Y_{\mathrm{e}, 3}=0.64$ and 0.66 , respectively (bottom panel in Figure 6). The end point of the $v p$-process appears to be at $A \sim 180\left({ }^{180} \mathrm{Ta}\right)$ in our explored cases. It should be noted that the wind termination also plays a crucial role as explored in Section 4.1. This is evident if we compare Figures 6 and 7, where the latter is the result for $r_{\mathrm{wt}}=\infty$. Without wind termination, more proton richness $\left(\Delta Y_{\mathrm{e}, 3} \sim 0.05\right)$ is required for a given $p$-nucleus to be produced, but with a substantially smaller production factor. The $p$-nuclei heavier than $A=140$ cannot be produced at all without wind termination (bottom panel in Figure 7).

We can understand the reason for the above result from Figure 8, which displays the snapshots of nucleosynthesis 

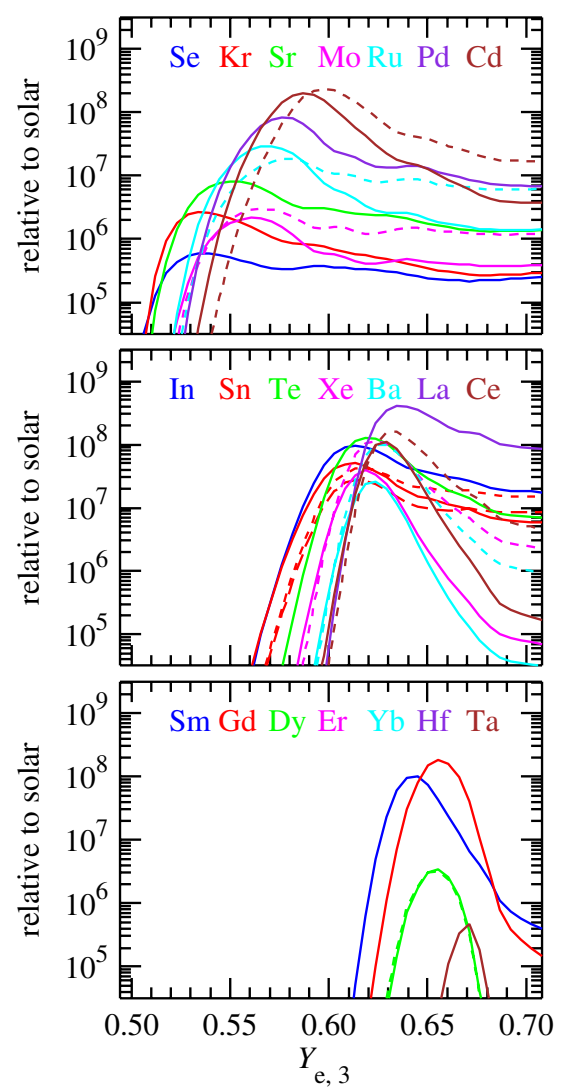

Figure 6. Nucleosynthetic $p$-abundances relative to their solar values (production factors) as a function of $Y_{\mathrm{e}, 3} . M_{\mathrm{ns}}, L_{v}$, and $r_{\mathrm{wt}}$ are kept to be their fiducial values (first line in Table 1). Each element is color coded with the solid, dashed, and long-dashed lines for the lightest, second-lightest, and third-lightest ${ }^{115} \mathrm{Sn}$ is only the case) isotopes, respectively (see the first column of Table 4 for the list of $p$-nuclei).

(A color version of this figure is available in the online journal.)

for selected cases on the nuclear chart when the temperature drops to $T_{9}=2$ (left) and 1 (right). Top, middle, and bottom panels are for the standard model, that with $Y_{\mathrm{e}, 9}$ replaced by $0.800\left(Y_{\mathrm{e}, 3}=0.655\right)$, and that with $Y_{\mathrm{e}, 9}$ and $r_{\mathrm{wt}}$ replaced by 0.800 and $\infty$ (without wind termination), respectively. In the standard model $\left(Y_{\mathrm{e}, 3}=0.550\right)$, the nuclear flow proceeds along the proton-drip line and encounters the proton-magic number $Z=50(A \sim 100-110)$. There are $\alpha$-unbound nuclei of ${ }^{106-108} \mathrm{Te}(Z=52)$ just above $Z=50$ along the proton-drip line, which is the end point of the classical rp-process (Schatz et al. 2001). This is why the $v p$-process stops at $A \sim 110$ for $Y_{\mathrm{e}, 3} \lesssim 0.6$.

As $Y_{\mathrm{e}, 3}$ exceeds 0.6 , radiative neutron capture becomes more important and competes with proton capture (Pruet et al. 2006; Wanajo 2006). This is due to the large amount of free protons $\left(Y_{\mathrm{p}} / Y_{\mathrm{h}}=1130\right.$ at $T_{9}=3$ for the middle panels of Figure 8; the last line in Table 1) that release free neutrons owing to neutrino capture $\left(\Delta_{\mathrm{n}}=94.2\right)$. As a result, the nuclear flow detours the end point of the classical rp-process $(N=54-56)$ at $Z=52$ toward the larger atomic number through the nuclei with $N>60$, as can be seen in Figure 9. The stagnation of the flow at the neutron-magic number $N=82$ in the middle panels of Figure 8 clearly shows the importance of neutron capture. The concentration of nuclei at $N=82$ leads to the large production factors of the $p$-nuclei with $A=130-150$ as seen in Figure 6. Note that the $p$-nuclide ${ }^{144} \mathrm{Sm}$ is located on the $N=82$ line.
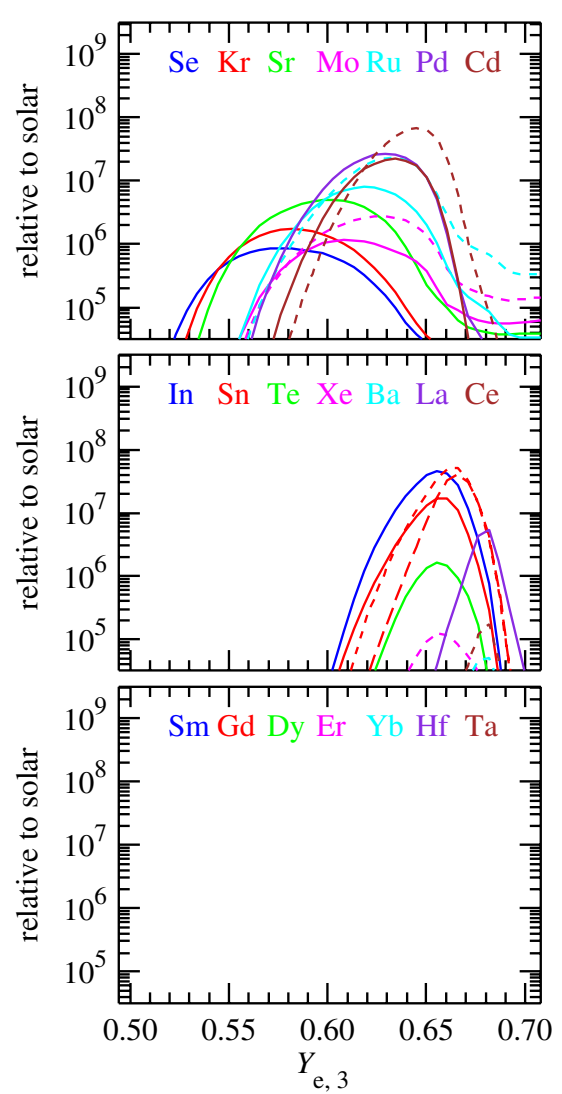

Figure 7. Same as Figure 6, but for the case without wind termination $\left(r_{\mathrm{wt}}=\infty\right)$. (A color version of this figure is available in the online journal.)

Beyond $N=82$, the increasing atomic number and the decreasing temperature inhibit further proton capture. Note that $n_{\bar{\nu}_{\mathrm{e}}}^{\prime} \sim 0.3 n_{\bar{\nu}_{\mathrm{e}}}$ in our explored models (see Equations (1) and (2)). Thus, neutron capture still continues at this stage. As a result, the nuclear flow approaches the $\beta$-stability line and finally enters to the neutron-rich region at $A \sim 160$ as seen in the middle-right panel of Figure 8. Without wind termination (but with the same parameters otherwise), however, the rapidly decreasing temperature does not allow the nuclear flow to reach $N=82$ as seen in the bottom panels of Figure 8. This is the reason for the inefficiency of producing heavy $p$-nuclei in Figure 7.

\section{UNCERTAINTIES IN NUCLEAR PHYSICS}

There have been continuing experimental works relevant to the $v p$-process (e.g., Weber et al. 2008; Hayakawa et al. 2010) since its discovery. However, we still rely upon theoretical or limited experimental estimates for the vast majority of nuclear reactions accompanied with the $v p$-process, which may suffer from uncertainties. There are also a number of isotopes without experimental mass measurements on the $v p$-process pathway (Weber et al. 2008).

The $v p$-process is unique in the following aspects, different from the classical rp-process. First is that the seed nuclei are directly formed from free nucleons (i.e., the primary process), while the classical rp-process needs CNO seeds. Thus, the triple- $\alpha$ process and some two-body reactions relevant to the breakout from the $p-p$ chain region $(A<12)$ play important roles for setting the proton-to-seed ratio $Y_{\mathrm{p}} / Y_{\mathrm{h}}$ (and thus $\Delta_{\mathrm{n}}$ ) at the beginning of the $v p$-process (Section 5.1). Second is the role 

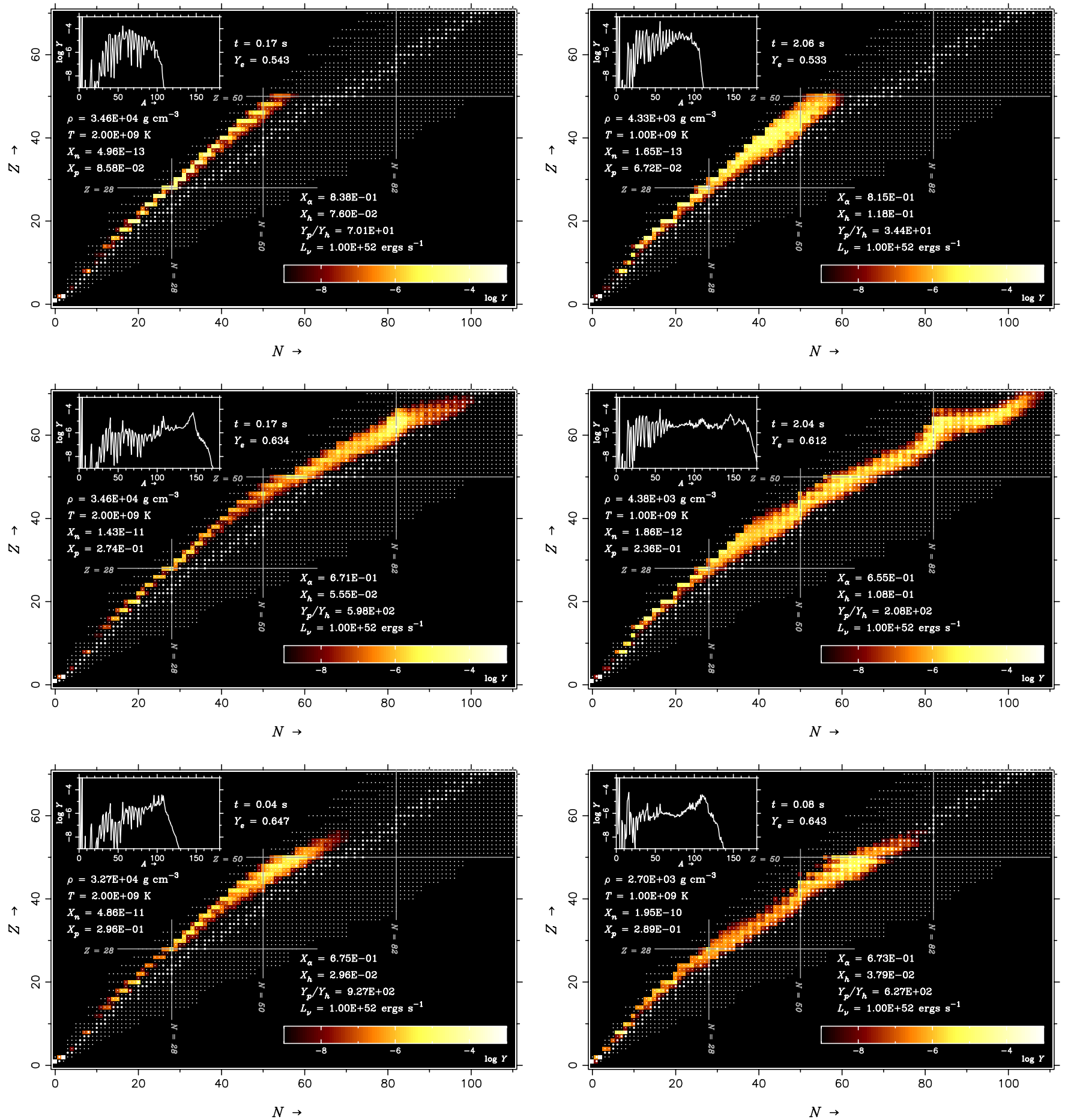

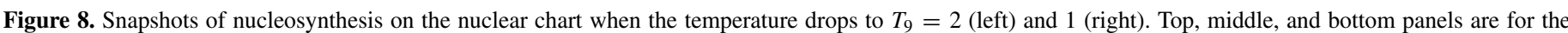
standard model (first line in Table 1), that with $Y_{\mathrm{e}, 9}$ replaced by $0.800\left(Y_{\mathrm{e}, 3}=0.655\right)$, and that with $Y_{\mathrm{e}, 9}$ and $r_{\mathrm{wt}}$ replaced by 0.800 and $\infty$ (without wind termination). The nucleosynthetic abundances are color coded. The species included in the reaction network are shown by dots (with the thick dots for the stable isotopes). The abundance distribution as a function of atomic mass number is shown in the inset of each panel.

(A color version of this figure is available in the online journal.)

of neutron capture, in particular of $(n, p)$ reactions on heavy nuclei in the proton-rich matter, which bypass the $\beta^{+}$-waiting points on the classical rp-process path (Section 5.2). Third, the $v p$-process path is limited to $Z \leqslant N$, where most of the nuclear masses of relevance are measurable (Weber et al. 2008; Section 5.3). This is an advantage compared with the classical rp-process that proceeds through even-even $Z=N$ nuclei with radiative proton capture to $Z>N$ isotopes (Brown et al. 2002).
In Section 5.1 and 5.2, we test the effect of uncertainties in some selected reactions by simply multiplying or dividing their original values by factors of 2 and 10 with the standard model (first line in Tables 1 and 3). All the explored results are listed in Table 3. In Section 5.3, the effect of new mass measurements by Weber et al. (2008) is discussed, along with possible uncertainties of other unmeasured nuclear masses on the $v p$-process pathway. 


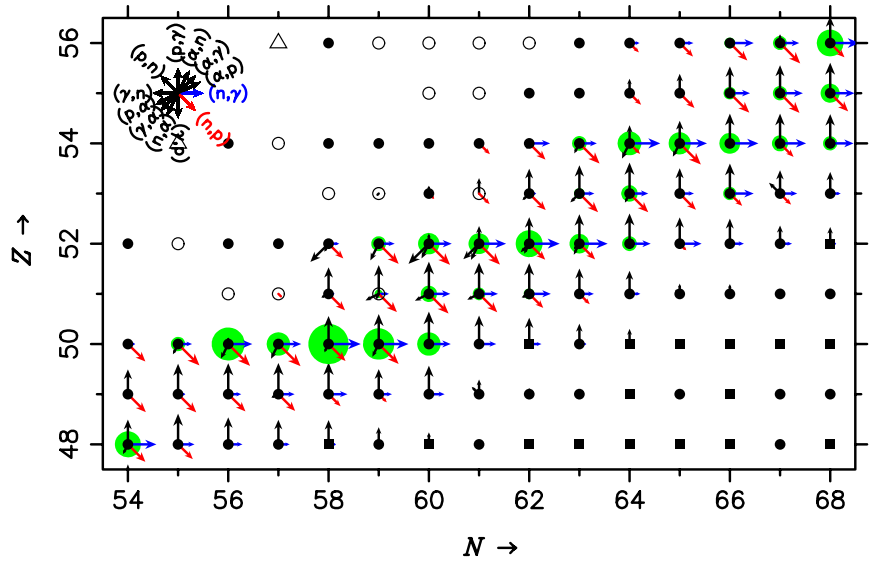

Figure 9. Nuclear flows (arrows) and the abundances (circles) near the end point of the classical rp-process $(Z=52)$ in logarithmic scale for the model with $Y_{\mathrm{e}, 3}=0.655$ (last line in Table 1) when the temperature decreases to $T_{9}=2$ (that corresponds to the middle-left panel in Figure 8). The nuclei included in the reaction network are denoted by squares (stable isotopes), filled circles (with measured masses of Audi et al. 2003), open circles (with extrapolated masses of Audi et al. 2003), and triangles (with the HFB-9 masses of Goriely et al. 2005). The flows by $\beta^{+}$-decays (not shown here) are negligible compared with those by $(n, p)$ reactions (red arrows). Radiative neutron capture (blue arrows) also plays a significant role.

(A color version of this figure is available in the online journal.)

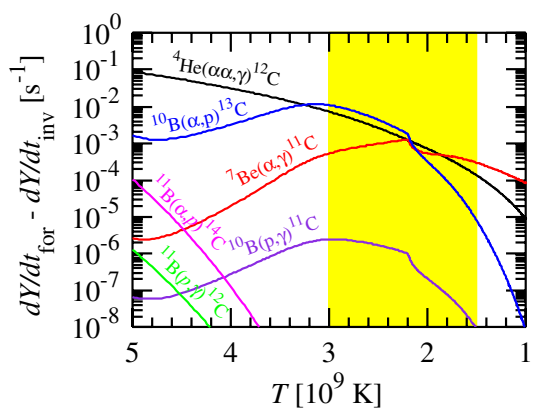

Figure 10. Nuclear flows for the reactions that bridge from $A<12$ (the $p-p$ chain region) to $A \geqslant 12$ as a function of temperature. The nuclear flow is defined as the deference of the time derivatives (per second) of abundance between the forward and inverse reactions for a given channel. The yellow band indicates the temperature range relevant to the $v p$-process $\left(T_{9}=1.5-3\right)$.

(A color version of this figure is available in the online journal.)

\subsection{Breakout from the $p-p$ Chain Region}

In Figure 10, the nuclear flows for the reactions that bridge from $A<12$ (the $p-p$ chain region) to $A \geqslant 12$ (the CNO region) are shown as a function of the temperature before $\left(T_{9}>3\right)$ and after $\left(T_{9}<3\right)$ the onset of the $v p$-process. The nuclear flows at $T_{9}=2.5$ for the relevant $N-Z$ region are also shown in Figure 11. Here, the nuclear flow is defined as the difference between the time derivatives of abundances for the forward and inverse reactions of a given channel. It is clear that, at a high temperature $\left(T_{9}>3\right)$, the triple- $\alpha$ process (with the rate of Caughlan \& Fowler 1988) plays a dominant role for the breakout from the $p-p$ chain region. We find, however, a couple of two-body reaction sequences ${ }^{7} \mathrm{Be}(\alpha, \gamma){ }^{11} \mathrm{C}(\alpha, p){ }^{14} \mathrm{~N}$ and ${ }^{7} \mathrm{Be}(\alpha, p){ }^{10} \mathrm{~B}(\alpha, p){ }^{13} \mathrm{C}$ compete with the triple- $\alpha$ process during the $v p$-process phase. ${ }^{9}$

\footnotetext{
$9 \quad{ }^{7} \mathrm{Be}(\alpha, p){ }^{10} \mathrm{~B}$ is an endothermic reaction. Because of its small (negative) $Q$-value of $-1.146 \mathrm{MeV}$ and the larger abundance of $\alpha$ particles, a small amount of ${ }^{10} \mathrm{~B}$ (that is immediately taken away by the subsequent $(\alpha, p)$ reaction) exists in the present case.
}

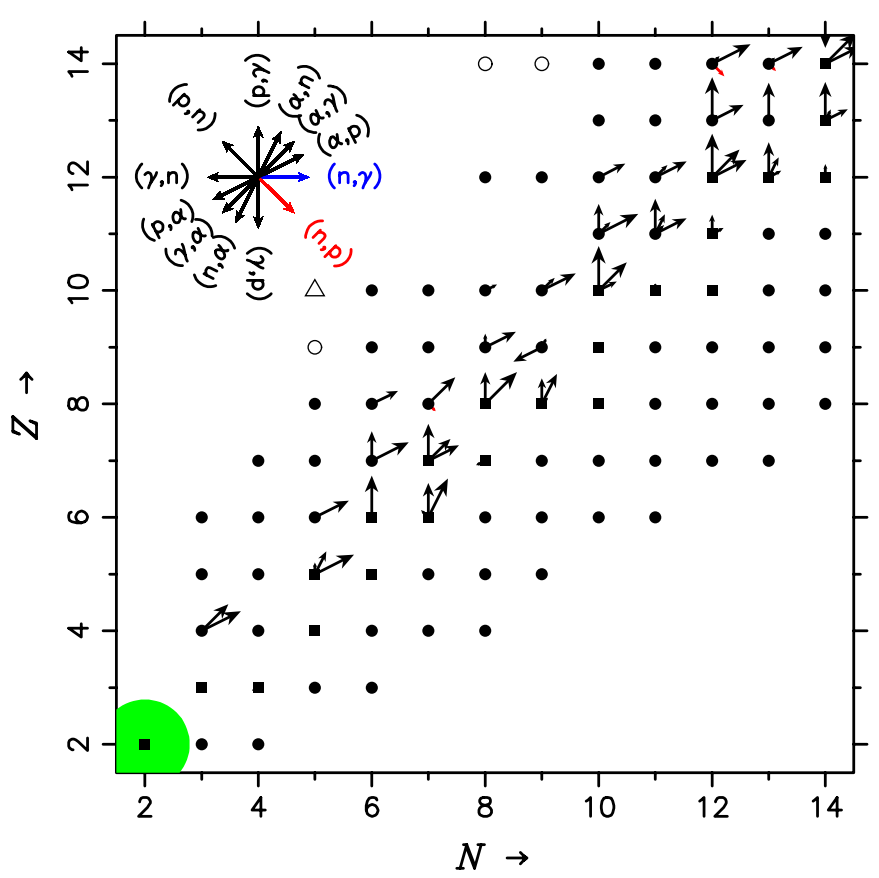

Figure 11. Same as Figure 9, but for the standard model when the temperature decreases to $T_{9}=2.5$ for a lighter $N-Z$ region. At this temperature, the nuclear flows through ${ }^{7} \mathrm{Be}(\alpha, \gamma){ }^{11} \mathrm{C}(\alpha, p){ }^{14} \mathrm{~N}$ and ${ }^{7} \mathrm{Be}(\alpha, p){ }^{10} \mathrm{~B}(\alpha, p){ }^{13} \mathrm{C}$ play dominant roles for the breakout from the $p-p$ chain region, along with the triple- $\alpha$ process (not shown here).

(A color version of this figure is available in the online journal.)

Table 2

Rates and Decay Timescales for Selected Reactions

\begin{tabular}{lcccc}
\hline \hline Species & $\begin{array}{c}\lambda_{2.5^{\mathrm{a}}} \\
\left(\mathrm{mol}^{-1} \mathrm{~cm}^{3} \mathrm{~s}^{-1}\right)\end{array}$ & $\begin{array}{c}\tau_{2.5}{ }^{\mathrm{a}} \\
(\mathrm{ms})\end{array}$ & $\begin{array}{c}\lambda_{2.0}{ }^{\mathrm{b}} \\
\left(\mathrm{mol}^{-1} \mathrm{~cm}^{3} \mathrm{~s}^{-1}\right)\end{array}$ & $\begin{array}{c}\tau_{2.0} \mathrm{~b} \\
(\mathrm{~ms})\end{array}$ \\
\hline $3 \alpha$ & $3.07 \times 10^{-10}$ & $8.32 \times 10^{4}$ & $3.86 \times 10^{-10}$ & $2.96 \times 10^{5}$ \\
${ }^{7} \mathrm{Be}(\alpha, \gamma)$ & 4.48 & $1.45 \times 10^{-2}$ & 1.29 & 0.107 \\
${ }^{7} \mathrm{Be}(\alpha, p)$ & $4.22 \times 10^{4}$ & $1.55 \times 10^{-6}$ & $5.89 \times 10^{3}$ & $2.34 \times 10^{-5}$ \\
${ }^{10} \mathrm{~B}(\alpha, p)$ & $5.76 \times 10^{5}$ & $1.13 \times 10^{-7}$ & $1.36 \times 10^{5}$ & $1.01 \times 10^{-6}$ \\
${ }^{10} \mathrm{~B}(p, \alpha)$ & $1.14 \times 10^{7}$ & $1.25 \times 10^{-8}$ & $6.04 \times 10^{6}$ & $5.59 \times 10^{-8}$ \\
${ }^{11} \mathrm{C}(\alpha, p)$ & $6.98 \times 10^{4}$ & $9.34 \times 10^{-7}$ & $1.61 \times 10^{4}$ & $8.58 \times 10^{-6}$ \\
${ }^{12} \mathrm{C}(p, \gamma)$ & $4.69 \times 10^{2}$ & $3.05 \times 10^{-4}$ & $5.11 \times 10^{2}$ & $6.59 \times 10^{-4}$ \\
${ }^{13} \mathrm{C}(\alpha, n)$ & $2.01 \times 10^{4}$ & $3.24 \times 10^{-6}$ & $1.27 \times 10^{4}$ & $1.09 \times 10^{-5}$ \\
${ }^{56} \mathrm{Ni}(n, p)$ & $1.61 \times 10^{8}$ & 11.3 & $1.28 \times 10^{8}$ & 454 \\
${ }^{60} \mathrm{Zn}(n, p)$ & $7.40 \times 10^{8}$ & 2.46 & $6.82 \times 10^{8}$ & 85.5 \\
${ }^{64} \mathrm{Ge}(n, p)$ & $6.85 \times 10^{8}$ & 2.65 & $6.15 \times 10^{8}$ & 94.8 \\
${ }^{68} \mathrm{Se}(n, p)$ & $9.38 \times 10^{8}$ & 1.94 & $8.12 \times 10^{8}$ & 71.8 \\
${ }^{72} \mathrm{Kr}(n, p)$ & $1.38 \times 10^{9}$ & 1.32 & $1.24 \times 10^{9}$ & 46.9 \\
${ }^{76} \mathrm{Sr}(n, p)$ & $1.81 \times 10^{9}$ & 1.00 & $1.69 \times 10^{9}$ & 34.6 \\
${ }^{80} \mathrm{Zr}(n, p)$ & $1.99 \times 10^{9}$ & 0.913 & $1.92 \times 10^{9}$ & 30.4 \\
${ }^{8} \mathrm{Mo}(n, p)$ & $1.44 \times 10^{9}$ & 1.26 & $1.31 \times 10^{9}$ & 44.5 \\
${ }^{96} \mathrm{Pd}(n, p)$ & $2.06 \times 10^{8}$ & 8.84 & $1.66 \times 10^{8}$ & 351 \\
\hline
\end{tabular}

Notes.

a Rates and decay timescales at $T_{9}=2.5\left(\rho=7.20 \times 10^{4} \mathrm{~g} \mathrm{~cm}^{-3}\right.$, $\left.X_{\mathrm{n}}=7.64 \times 10^{-12}, X_{\mathrm{p}}=0.0970, X_{\alpha}=0.852\right)$.

b Rates and decay timescales at $T_{9}=2.0\left(\rho=3.46 \times 10^{4} \mathrm{~g} \mathrm{~cm}^{-3}\right.$, $\left.X_{\mathrm{n}}=4.96 \times 10^{-13}, X_{\mathrm{p}}=0.0858, X_{\alpha}=0.838\right)$.

Table 2 lists the reaction rates and decay timescales for the relevant isotopes at $T_{9}=2.5$ and 2.0. It is clear that ${ }^{7} \mathrm{Be}(\alpha, \gamma)^{11} \mathrm{C}$, four orders of magnitude slower than ${ }^{11} \mathrm{C}(\alpha, p){ }^{14} \mathrm{~N}$, governs the former sequence. For the latter, ${ }^{10} \mathrm{~B}(\alpha, p){ }^{13} \mathrm{C}$, although a factor of 10 smaller than ${ }^{7} \mathrm{Be}(\alpha, p){ }^{10} \mathrm{~B}$, mainly controls the reaction flow, which takes away nuclear abundances from ${ }^{10} \mathrm{~B}$ formed by the endothermic reaction. 

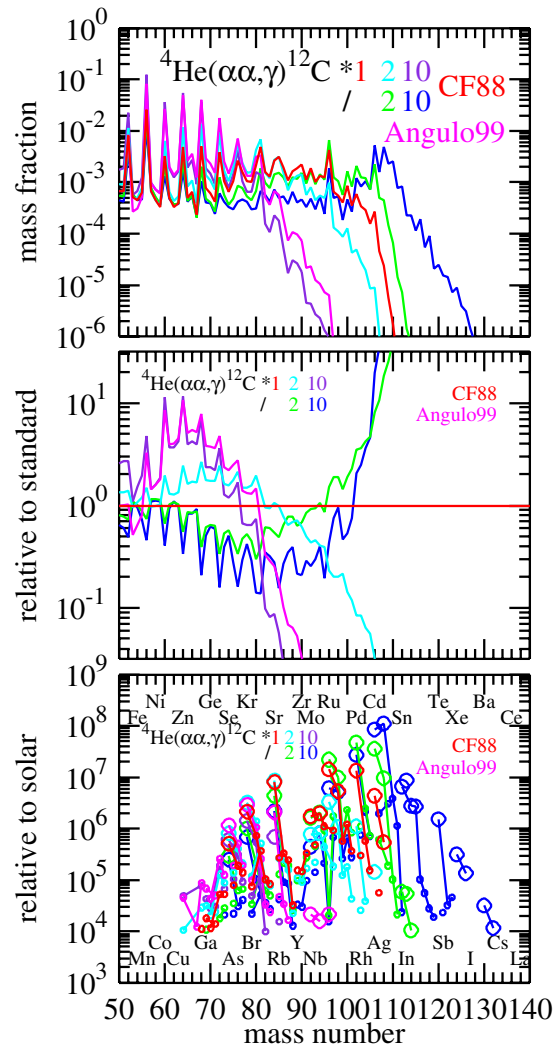

Figure 12. Same as Figure 2, but for variations on the triple- $\alpha$ rate. (A color version of this figure is available in the online journal.)

Figure 10 shows that ${ }^{7} \mathrm{Be}(\alpha, \gamma){ }^{11} \mathrm{C}$ and ${ }^{10} \mathrm{~B}(\alpha, p){ }^{13} \mathrm{C}$ exhibit similar roles to triple- $\alpha$ in the temperature range relevant to the $v p$-process. Therefore, we select these three reactions for the sensitivity tests. Note that the unstable isotope ${ }^{11} \mathrm{C}$ produced is followed by ${ }^{11} \mathrm{C}(\alpha, p){ }^{14} \mathrm{~N}$ (see Hayakawa et al. 2010 for a recent experimental evaluation of this rate) before decaying back to ${ }^{11} \mathrm{~B}$.

All the data of these three reactions, from Wagoner (1969, for $\left.{ }^{10} \mathrm{~B}(\alpha, p){ }^{13} \mathrm{C}\right)$ and Caughlan \& Fowler (1988, for the remainder) in the REACLIB compilation, are based on experimental information of single resonance states. Contribution from (possible) resonances at higher excitation energies could thus sizably change these rates. As an example, the triple- $\alpha$ rate of Angulo et al. (1999), which includes contribution from the $9.2 \mathrm{MeV}$ $2^{+}$state that is predicted theoretically, leads to a factor of $2-10$ higher values (for the temperature range relevant to seed production, $T_{9}=7-3$ ) than that of Caughlan \& Fowler (1988) based on the single 7.6 $\mathrm{MeV} \mathrm{0}^{+}$(Hoyle) state. Recent experimental works did not confirm the presence of the $9.2 \mathrm{MeV}$ state, but other levels in this energy region as well as those at higher energies might contribute to this rate (Austin 2005; Diget et al. 2005, 2009).

The result of sensitivity tests for the triple- $\alpha$ rate is shown in Figure 12, where the forward and inverse rates are multiplied or divided by factors of 2 and 10 . We find substantial changes in the production of $p$-nuclei with $A \sim 100-110$ for a factor of two variation on the rate, and more drastic changes for a factor of 10 variation. It can be mainly attributed to the resulting protonto-seed ratio $Y_{\mathrm{p}} / Y_{\mathrm{h}}\left(\right.$ at $T_{9}=3$ ) and thus $\Delta_{\mathrm{n}}$ (third and fourth lines in Table 3). Note that $n_{\bar{\nu}_{\mathrm{e}}}(=0.0834$; first line in Table 1) remains the same for all the cases here. A larger triple- $\alpha$ rate leads to a more efficient seed production and thus smaller $Y_{\mathrm{p}} / Y_{\mathrm{h}}$

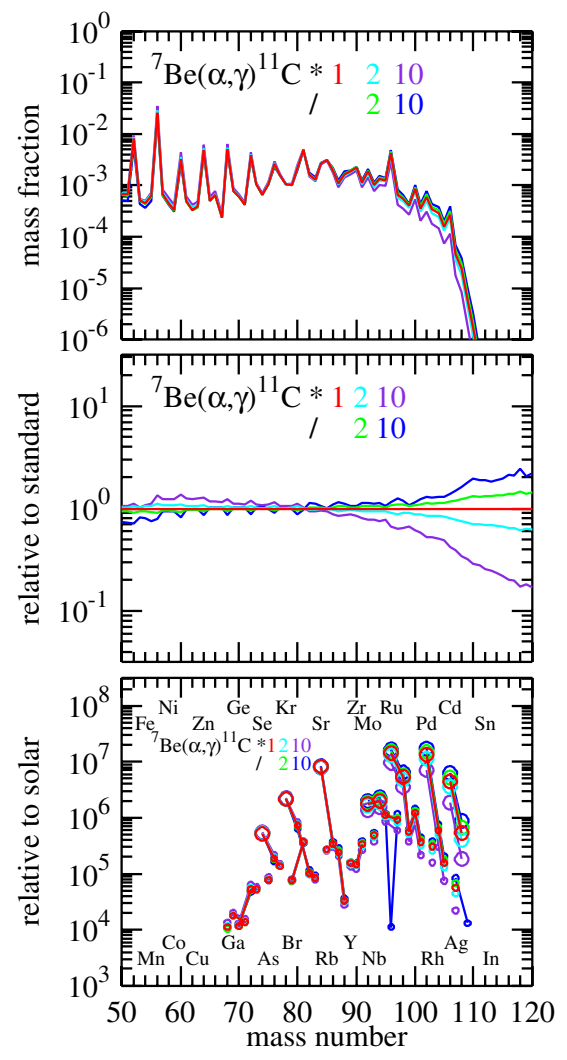

Figure 13. Same as Figure 2, but for variations on the ${ }^{7} \mathrm{Be}(\alpha, \gamma){ }^{11} \mathrm{C}$ rate. (A color version of this figure is available in the online journal.)

and $\Delta_{\mathrm{n}}$. A larger rate during the $v p$-process phase $\left(T_{9}=1.5-3\right)$ also yields more carbon and other intermediate-mass nuclei that act as proton poison. As a result, efficiency of the $v p$-process for heavy element synthesis decreases. The same interpretation is applicable to the opposite case with a smaller rate.

We find that a replacement of the triple- $\alpha$ rate by that of Angulo et al. (1999) inhibits production of $p$-nuclei for $A>80$ (Figure 12). In fact, the net effect of including the $9.2 \mathrm{MeV}$ state (not confirmed by recent experiments) by Angulo et al. (1999) corresponds to the rate of Caughlan \& Fowler (1988) multiplied by a factor of 10 . This demonstrates the importance of future re-evaluations of (possible) contribution from higher levels than the $7.64 \mathrm{MeV}$ state in ${ }^{12} \mathrm{C}$.

Figures 13 and 14 show the result for ${ }^{7} \operatorname{Be}(\alpha, \gamma){ }^{11} \mathrm{C}$ and ${ }^{10} \mathrm{~B}(\alpha, p){ }^{13} \mathrm{C}$. We find non-negligible differences in the $p$-abundances with $A \sim 100-110$, although the impact is much smaller than that for triple- $\alpha$. Note that a larger rate has a stronger impact than a smaller rate (middle panels). This is a consequence of the fact that the larger rate of a given channel increases the total efficiency for the breakout from the $p-p$ chain region, while the other two channels are still active for the smaller rate (see Figure 10).

${ }^{7} \mathrm{Be}(\alpha, \gamma){ }^{11} \mathrm{C}$ competes with triple- $\alpha$ only during the late phase of the $v p$-process $\left(T_{9} \lesssim 2\right.$; Figure 10$)$. A larger rate during this phase leads to more production of intermediate-mass nuclei that act as proton poison. A variation of this rate does not substantially affect $Y_{\mathrm{p}} / Y_{\mathrm{h}}\left(\right.$ at $\left.T_{9}=3\right)$ and $\Delta_{\mathrm{n}}$ at the onset of $v p$-processing (Table 3 ). ${ }^{10} \mathrm{~B}(\alpha, p){ }^{13} \mathrm{C}$ however competes with triple- $\alpha$ at $T_{9} \sim 2-3.5$ (Figure 10). Hence, a variation on the rate also affects $Y_{\mathrm{p}} / Y_{\mathrm{h}}$ at the beginning of $\nu p$-processing and $\Delta_{\mathrm{n}}$ (Table 3). 
Table 3

Results for the Changes of Reaction Rates

\begin{tabular}{|c|c|c|c|c|c|c|c|}
\hline Reaction & Factor & $Y_{\mathrm{p}} / Y_{\mathrm{h}}^{\mathrm{a}}$ & $\Delta_{n}^{\mathrm{b}}$ & $\log f_{\max } \mathrm{c}$ & $\operatorname{nuc}(f)^{\mathrm{d}}$ & $\operatorname{nuc}(A)^{\mathrm{e}}$ & Fig. \\
\hline Standard & 1.00 & 124 & 10.3 & 7.16 & ${ }^{96} \mathrm{Ru}$ & ${ }^{106} \mathrm{Cd}$ & All \\
\hline $3 \alpha$ & $1.00^{\mathrm{f}}$ & 25.6 & 2.14 & 6.47 & ${ }^{78} \mathrm{Kr}$ & ${ }^{84} \mathrm{Sr}$ & 12 \\
\hline $3 \alpha$ & 2.00 & 73.5 & 6.13 & 6.93 & ${ }^{84} \mathrm{Sr}$ & ${ }^{102} \mathrm{Pd}$ & 12 \\
\hline $3 \alpha$ & 10.0 & 25.2 & 2.10 & 6.15 & ${ }^{78} \mathrm{Kr}$ & ${ }^{84} \mathrm{Sr}$ & 12 \\
\hline $3 \alpha$ & $1 / 2.00$ & 204 & 17.0 & 7.67 & ${ }^{102} \mathrm{Pd}$ & ${ }^{108} \mathrm{Cd}$ & 12 \\
\hline $3 \alpha$ & $1 / 10.0$ & 482 & 40.2 & 8.04 & ${ }^{108} \mathrm{Cd}$ & ${ }^{108} \mathrm{Cd}$ & 12 \\
\hline $3 \alpha$ & $1 / 100$ & 719 & 60.0 & 8.02 & ${ }^{108} \mathrm{Cd}$ & ${ }^{120} \mathrm{Te}$ & 12 \\
\hline${ }^{7} \operatorname{Be}(\alpha, \gamma)$ & 2.00 & 124 & 10.3 & 7.11 & ${ }^{96} \mathrm{Ru}$ & ${ }^{106} \mathrm{Cd}$ & 13 \\
\hline${ }^{7} \operatorname{Be}(\alpha, \gamma)$ & 10.0 & 122 & 10.2 & 6.98 & ${ }^{96} \mathrm{Ru}$ & ${ }^{106} \mathrm{Cd}$ & 13 \\
\hline${ }^{7} \operatorname{Be}(\alpha, \gamma)$ & 100 & 117 & 9.76 & 6.89 & ${ }^{84} \mathrm{Sr}$ & ${ }^{106} \mathrm{Cd}$ & 13 \\
\hline${ }^{7} \operatorname{Be}(\alpha, \gamma)$ & $1 / 2.00$ & 124 & 10.3 & 7.19 & ${ }^{96} \mathrm{Ru}$ & ${ }^{106} \mathrm{Cd}$ & 13 \\
\hline${ }^{7} \operatorname{Be}(\alpha, \gamma)$ & $1 / 10.0$ & 124 & 10.3 & 7.24 & ${ }^{102} \mathrm{Pd}$ & ${ }^{106} \mathrm{Cd}$ & 13 \\
\hline${ }^{10} \mathrm{~B}(\alpha, p)$ & 2.00 & 119 & 9.92 & 7.09 & ${ }^{96} \mathrm{Ru}$ & ${ }^{106} \mathrm{Cd}$ & 14 \\
\hline${ }^{10} \mathrm{~B}(\alpha, p)$ & 10.0 & 112 & 9.34 & 6.96 & ${ }^{96} \mathrm{Ru}$ & ${ }^{106} \mathrm{Cd}$ & 14 \\
\hline${ }^{10} \mathrm{~B}(\alpha, p)$ & $1 / 2.00$ & 129 & 10.8 & 7.23 & ${ }^{102} \mathrm{Pd}$ & ${ }^{106} \mathrm{Cd}$ & 14 \\
\hline${ }^{10} \mathrm{~B}(\alpha, p)$ & $1 / 10.0$ & 135 & 11.3 & 7.35 & ${ }^{102} \mathrm{Pd}$ & ${ }^{106} \mathrm{Cd}$ & 14 \\
\hline${ }^{56} \mathrm{Ni}(n, p)$ & 2.00 & 124 & 10.3 & 7.01 & ${ }^{96} \mathrm{Ru}$ & ${ }^{106} \mathrm{Cd}$ & 16 \\
\hline${ }^{56} \mathrm{Ni}(n, p)$ & 10.0 & 124 & 10.3 & 7.02 & ${ }^{84} \mathrm{Sr}$ & ${ }^{102} \mathrm{Pd}$ & 16 \\
\hline${ }^{56} \mathrm{Ni}(n, p)$ & $1 / 2.00$ & 124 & 10.3 & 7.45 & ${ }^{102} \mathrm{Pd}$ & ${ }^{106} \mathrm{Cd}$ & 16 \\
\hline${ }^{56} \mathrm{Ni}(n, p)$ & $1 / 10.0$ & 124 & 10.3 & 7.92 & ${ }^{106} \mathrm{Cd}$ & ${ }^{108} \mathrm{Cd}$ & 16 \\
\hline${ }^{60} \mathrm{Zn}(n, p)$ & 2.00 & 124 & 10.3 & 7.15 & ${ }^{96} \mathrm{Ru}$ & ${ }^{106} \mathrm{Cd}$ & 19 \\
\hline${ }^{60} \mathrm{Zn}(n, p)$ & 10.0 & 124 & 10.3 & 7.15 & ${ }^{96} \mathrm{Ru}$ & ${ }^{106} \mathrm{Cd}$ & 19 \\
\hline${ }^{60} \mathrm{Zn}(n, p)$ & $1 / 2.00$ & 124 & 10.3 & 7.22 & ${ }^{102} \mathrm{Pd}$ & ${ }^{106} \mathrm{Cd}$ & 19 \\
\hline${ }^{60} \mathrm{Zn}(n, p)$ & $1 / 10.0$ & 124 & 10.3 & 7.51 & ${ }^{102} \mathrm{Pd}$ & ${ }^{108} \mathrm{Cd}$ & 19 \\
\hline${ }^{64} \mathrm{Ge}(n, p)$ & 2.00 & 124 & 10.3 & 7.16 & ${ }^{96} \mathrm{Ru}$ & ${ }^{106} \mathrm{Cd}$ & 20 \\
\hline${ }^{64} \mathrm{Ge}(n, p)$ & 10.0 & 124 & 10.3 & 7.16 & ${ }^{96} \mathrm{Ru}$ & ${ }^{106} \mathrm{Cd}$ & 20 \\
\hline${ }^{64} \mathrm{Ge}(n, p)$ & $1 / 2.00$ & 124 & 10.3 & 7.19 & ${ }^{102} \mathrm{Pd}$ & ${ }^{106} \mathrm{Cd}$ & 20 \\
\hline${ }^{64} \mathrm{Ge}(n, p)$ & $1 / 10.0$ & 124 & 10.3 & 7.41 & ${ }^{102} \mathrm{Pd}$ & ${ }^{108} \mathrm{Cd}$ & 20 \\
\hline
\end{tabular}

Notes.

a Proton-to-seed ratio at $T_{9}=3$.

b $\Delta_{n}$ at $T_{9}=3$.

${ }^{c}$ Maximum production factor.

d Nucleus at $f=f_{\text {max }}$.

e Nucleus at the largest $A$ with $f>f_{\max } / 10$.

f Triple- $\alpha$ rate from Angulo et al. (1999).

\section{2. $(n, p)$ Reactions on Heavy Nuclei}

The $v p$-process starts at $T_{9} \sim 3$ from the seed nucleus ${ }^{56} \mathrm{Ni}$, which is formed from free nucleons earlier. During the $v p$-process, the $(n, p)$ reactions play an important role for determining the nuclear flows. Figure 15 shows the nuclear flows starting from ${ }^{56} \mathrm{Ni}(Z=N=28)$ up to ${ }^{80} \mathrm{Zr}(Z=N=40)$. The isotopes included in the reaction network are denoted by squares (stable isotopes), filled circles (with measured masses of Audi et al. 2003), open circles (with extrapolated masses of Audi et al. 2003), and triangles (with the HFB-9 masses of Goriely et al. 2005). We find that the nuclear flow of the $v p$-process proceeds through even-even $Z=N$ isotopes up to $Z=N=40$ as in the classical rp-process. All the nuclear masses on the $v p$-process path (up to $Z=N=40$ ), which determine the abundance distribution for given isotones, were measured by experiments (Audi et al. 2003). ${ }^{10}$

Currently, there are no experiment-based estimates for the $(n, p)$ reactions on proton-rich isotopes along the $v p$-process path. We rely upon the theoretically predicted Hauser-Feshbach rates, which are generally considered to involve uncertainties up to a factor of a few (this reduces to $\sim 40 \%$ if the nuclear levels

\footnotetext{
10 We do not take the mirror-mass evaluations of Brown et al. (2002) for $Z>N$ nuclei into account, as the $v p$-process path is limited to the $Z \leqslant N$ region (except for a flow to ${ }^{59} \mathrm{Zn}$ but with measured masses; Figure 15).
}
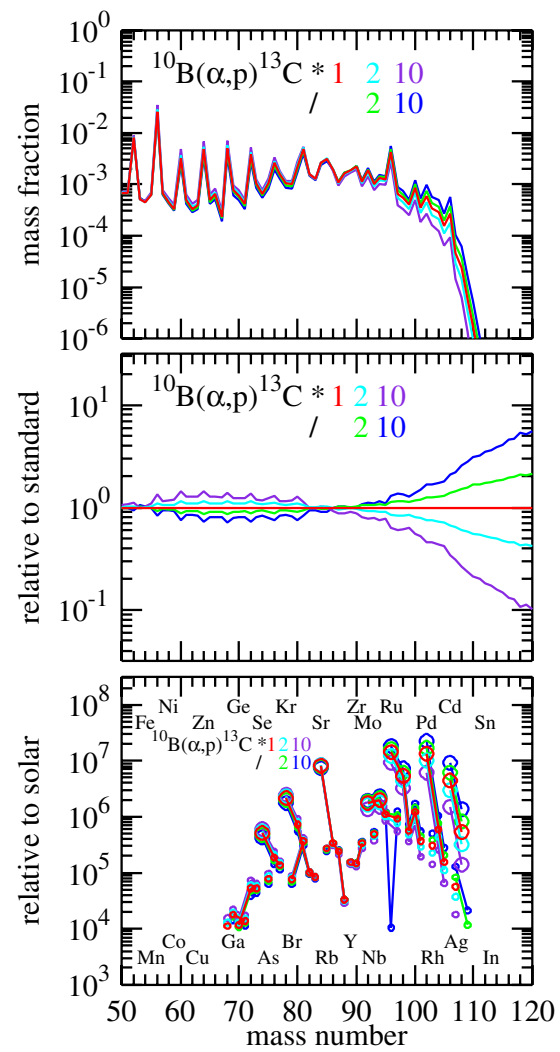

Figure 14. Same as Figure 2, but for variations on the ${ }^{10} \mathrm{~B}(\alpha, p){ }^{13} \mathrm{C}$ rate. (A color version of this figure is available in the online journal.)

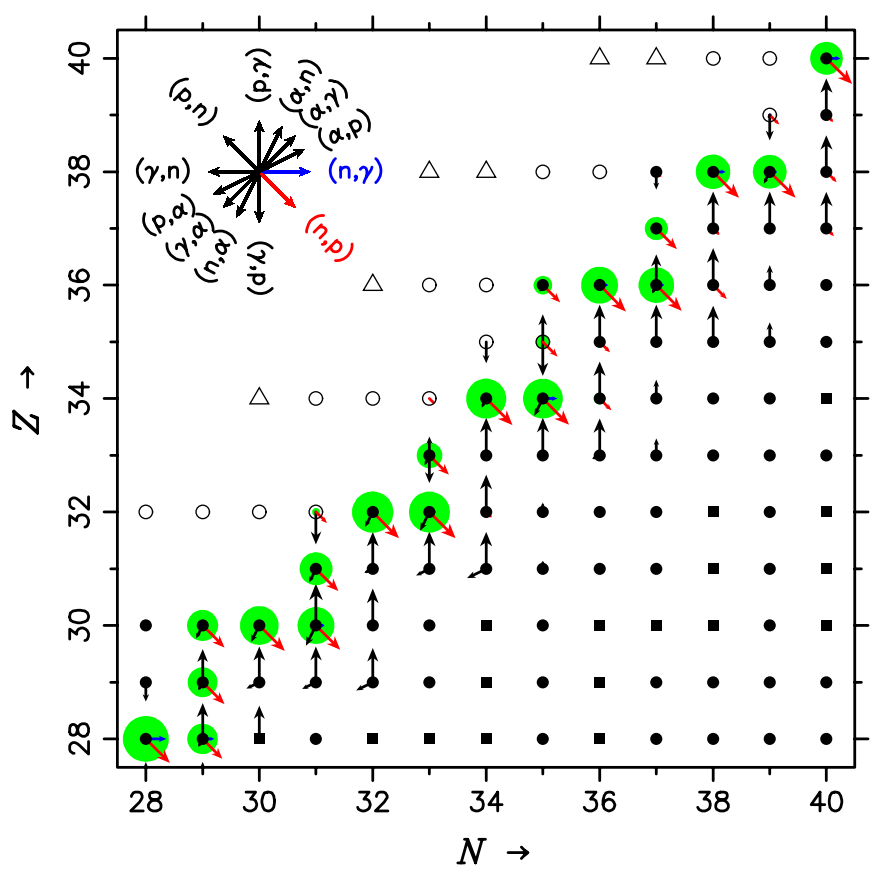

Figure 15. Same as Figure 9, but for the standard model when the temperature decreases to $T_{9}=2.0$.

(A color version of this figure is available in the online journal.)

are well determined and the level densities are large enough, Rauscher et al. 1997). Rauscher (2010) also finds sizable shifts of effective energy windows for $(n, p)$ at high temperature, which might modify these rates. In this subsection, therefore, 


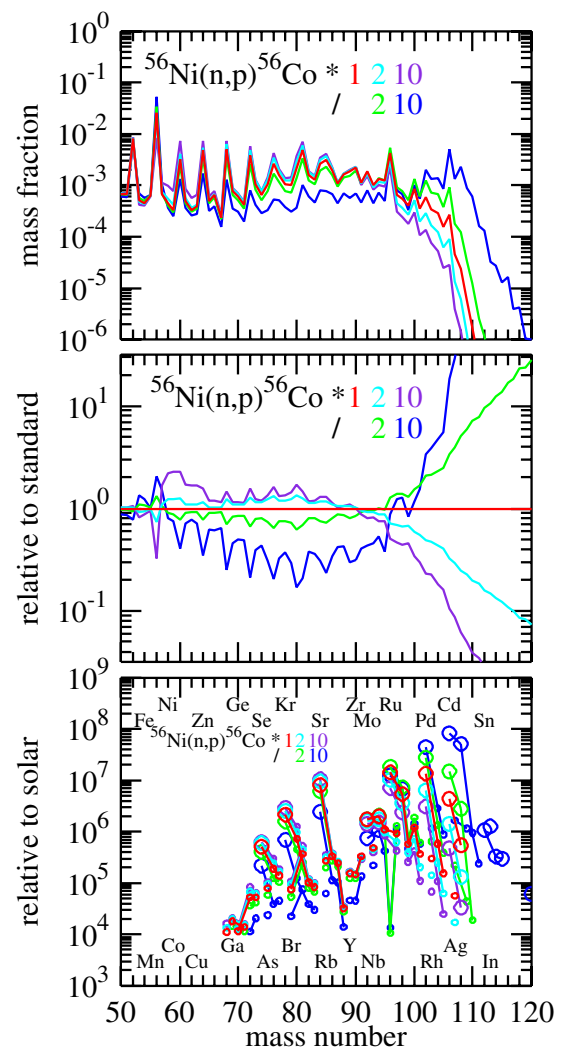

Figure 16. Same as Figure 2, but for variations on the ${ }^{56} \mathrm{Ni}(n, p){ }^{56} \mathrm{Co}$ rate. (A color version of this figure is available in the online journal.)

the sensitivity tests for $(n, p)$ reactions are made with factors of 2 and 10 variations as in Section 5.1.

We here pick up three $(n, p)$ reactions starting from the seed nuclei along the $v p$-process path, i.e., on ${ }^{56} \mathrm{Ni},{ }^{60} \mathrm{Zn}$, and ${ }^{64} \mathrm{Ge}$. The last one, ${ }^{64} \mathrm{Ge}$, is the first $\beta^{+}$-waiting point nucleus encountered in the classical rp-process path. Note that the variations on these rates do not affect $Y_{\mathrm{p}} / Y_{\mathrm{h}}$ at the onset of the $v p$-process nor $\Delta_{\mathrm{n}}$ (Table 3). All these rates are from theoretical estimates in BRUSLIB (Aikawa et al. 2005) making use of experimental masses (Audi et al. 2003). Our test calculations with the $(n, p)$ rates replaced by those in the REACLIB compilation (Rauscher et al. 2002) are in reasonable agreement (within factor of a few) with our standard case (see also Wanajo et al. 2009).

We find a remarkable change in the $p$-abundances with $A \sim 110$ by a factor of 10 with only a factor of two variation on ${ }^{56} \mathrm{Ni}(n, p){ }^{56} \mathrm{Co}$ (Figure 16 and Table 3$)$. This demonstrates that the $(n, p)$ reaction on the first $(n, p)$-waiting nucleus ${ }^{56} \mathrm{Ni}$ plays a key role for the progress of nuclear flows.

It should be noted that a smaller rate leads to more efficient $v p$-processing as can be seen in the bottom panel of Figure 16 and in Table 3 (see $f_{\max }$ and $A_{\max }$ ). The reason can be explained as follows: Figure 17 extends the nuclear flows in Figure 15. We find that the $v p$-process path proceeds through even-even $Z=N$ isotopes and deviates from ${ }^{84}$ Mo $(Z=N=42)$ toward $Z<N$, reaching ${ }^{96} \mathrm{Pd}$ on the $N=50$ shell closure. These isotopes have large abundances during the whole $v p$ process phase, as can be seen in Figures 15 and 17 (filled green circles). The top panel of Figure 16 shows that the abundances with $A=60,64,68,72,76,80$, and 84 are similar to that of $A=96$. Table 2 lists the $(n, p)$ rates and decay timescales

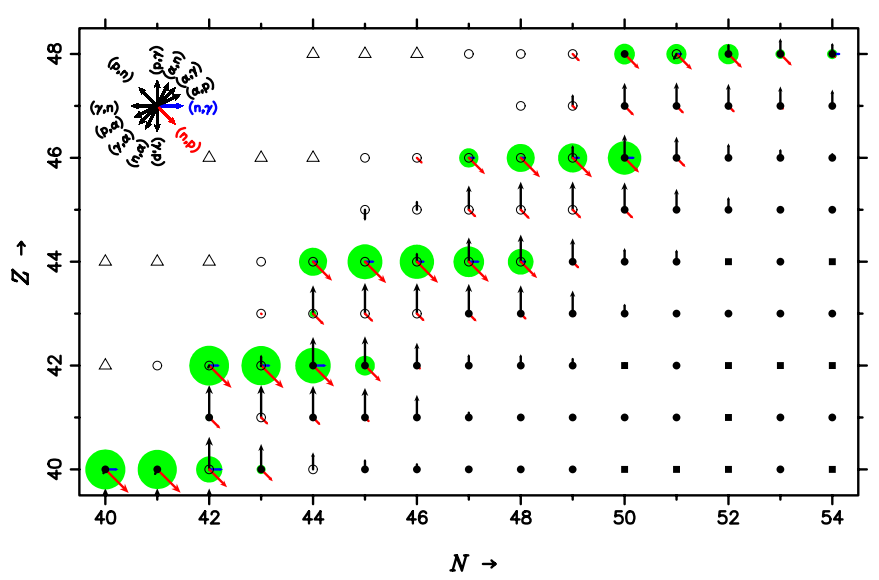

Figure 17. Same as Figure 9, but for the standard model when the temperature decreases to $T_{9}=2.0$.

(A color version of this figure is available in the online journal.)

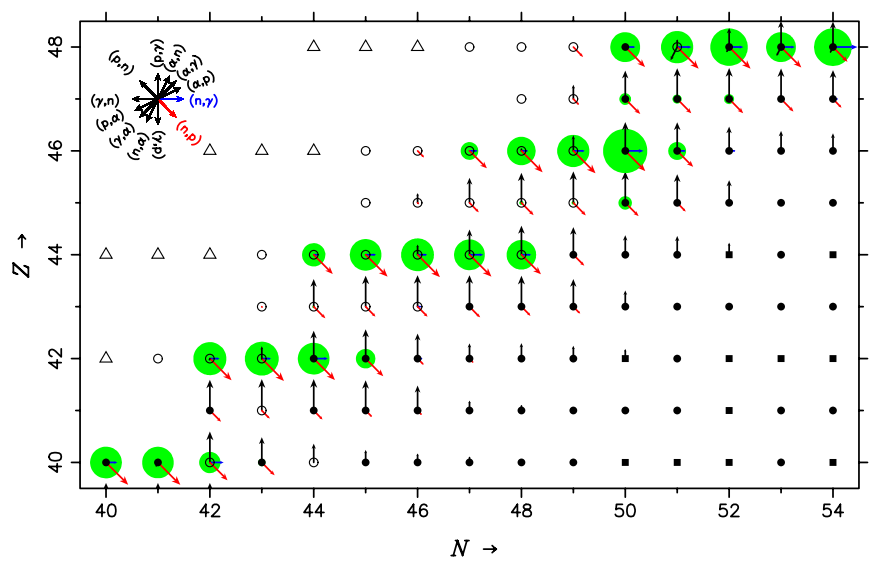

Figure 18. Same as Figure 17, but for the model with the ${ }^{56} \mathrm{Ni}(n, p)^{56} \mathrm{Co}$ rate and its inverse reduced by a factor of $10\left(\right.$ at $\left.T_{9}=2.0\right)$.

(A color version of this figure is available in the online journal.)

for the corresponding isotopes at $T_{9}=2.5$ and 2.0. The $(n, p)$ rates on ${ }^{56} \mathrm{Ni}$ and ${ }^{96} \mathrm{Pd}$, neutron magic nuclei on $N=28$ and 50 , respectively, are a factor of 4-10 times smaller than the others. This indicates that the free neutrons created by neutrino capture $\left(\Delta_{\mathrm{n}}\right)$ are preferentially consumed by the even- $Z$ nuclei with $30 \leqslant Z \leqslant 42$ (that act as neutron poisons), rather than by ${ }^{96} \mathrm{Pd}$.

A reduction of the ${ }^{56} \mathrm{Ni}(n, p)$ rate (by a factor of 2 or 10) reduces the abundances of these neutron poisons by a similar factor ( $A \sim 60-90$; Figure 16, middle panel). Despite this, the abundance of ${ }^{96} \mathrm{Pd}$ does not decrease (even increases). This is due to the faster $(n, p)$ rates for the $Z=N(=30-42)$ nuclei, causing the nuclear flows from ${ }^{56} \mathrm{Ni}$ to immediately reach ${ }^{96} \mathrm{Pd}$ and to stagnate there. As a result, a larger number of free neutrons become available for the ${ }^{96} \mathrm{Pd}(n, p)$ reaction.

The nuclear flows for the ${ }^{56} \mathrm{Ni}(n, p)$ rate reduced by a factor of 10 are shown in Figure 18. Smaller abundances of $Z=N$ nuclei ${ }^{80} \mathrm{Zr}$ and ${ }^{84} \mathrm{Mo}$ can be seen, which leads to the larger flows beyond $N=50$ through ${ }^{96} \mathrm{Pd}$. This clearly demonstrates that ${ }^{96} \mathrm{Pd}$ plays a role as a "second seed nucleus" for producing nuclei heavier than $A=96$. In short, a reduction of the ${ }^{56} \mathrm{Ni}(n, p)$ rate increases the number of free neutrons available for the second seed nuclei of ${ }^{96} \mathrm{Pd}$.

Figures 19 and 20 show the results for the second and third $(n, p)$-waiting nuclei, ${ }^{60} \mathrm{Zn}$ and ${ }^{64} \mathrm{Ge}$ (the first $\beta^{+}$-waiting 


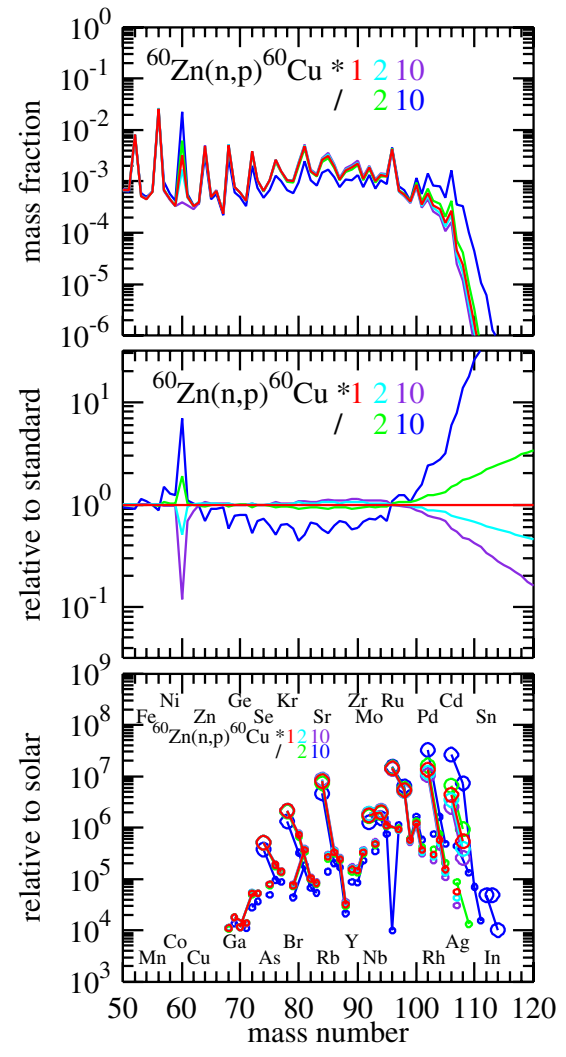

Figure 19. Same as Figure 2, but for variations on the ${ }^{60} \mathrm{Zn}(n, p){ }^{60} \mathrm{Cu}$ rate (A color version of this figure is available in the online journal.)

nucleus on the classical rp-process). The variations on these rates also lead to visible changes in the nucleosynthetic $p$-abundances, being however less prominent than in the case of ${ }^{56} \mathrm{Ni}$. Note that a reduced $(n, p)$ rate leads to a larger impact on the nucleosynthetic $p$-abundances than an increased value of this rate. This is due to the fact that the $(n, p)$ reaction on ${ }^{56} \mathrm{Ni}$ is substantially slower than those on ${ }^{60} \mathrm{Zn}$ and ${ }^{64} \mathrm{Ge}$ (Table 2), where the strength of the nuclear flow is limited by the former reaction.

\subsection{Nuclear Masses on the vp-Process Pathway}

Nuclear masses on the nucleosynthetic path are fundamental for all the relevant nuclear (or weak) processes. In particular, the flow strength of radiative proton capture during $v p$-processing $\left(T_{9}=3-1.5\right)$ is mostly determined from proton separation energies, where $(p, \gamma) \leftrightarrow(\gamma, p)$ is generally faster than $(n, p)$ and $(n, \gamma)$ and thus in a QSE. This explains the concentration of abundances on even- $Z$ isotopes in Figures 8 (left panel; in particular for $Z \leqslant 50), 9,15,17$, and 18 .

There are a number of isotopes without measured masses in the compilation of Audi et al. (2003) for $40 \leqslant Z \leqslant 50$ (denoted by open circles in Figures 17 and 18), including the parent nuclei of light $p$-nuclei, ${ }^{84} \mathrm{Sr},{ }^{92,94} \mathrm{Mo}$, and ${ }^{96,98} \mathrm{Ru}$. Pruet et al. (2006) noted that the unmeasured masses of ${ }^{92} \mathrm{Ru}$ and ${ }^{93} \mathrm{Rh}$ (i.e., the proton separation energy of ${ }^{93} \mathrm{Rh}$ ) on the $N=48$ isotones are crucial for determining the ratio of ${ }^{92} \mathrm{Mo} /{ }^{94} \mathrm{Mo}$.

Recently, Weber et al. (2008) obtained precision measurements of a number of nuclear masses along the $v p$-process pathway, including those of ${ }^{92} \mathrm{Ru}$ and ${ }^{93} \mathrm{Rh}$. Here, we present the nucleosynthetic result with inclusion of their new masses, denoted by star symbols in Figure 21, with our standard model
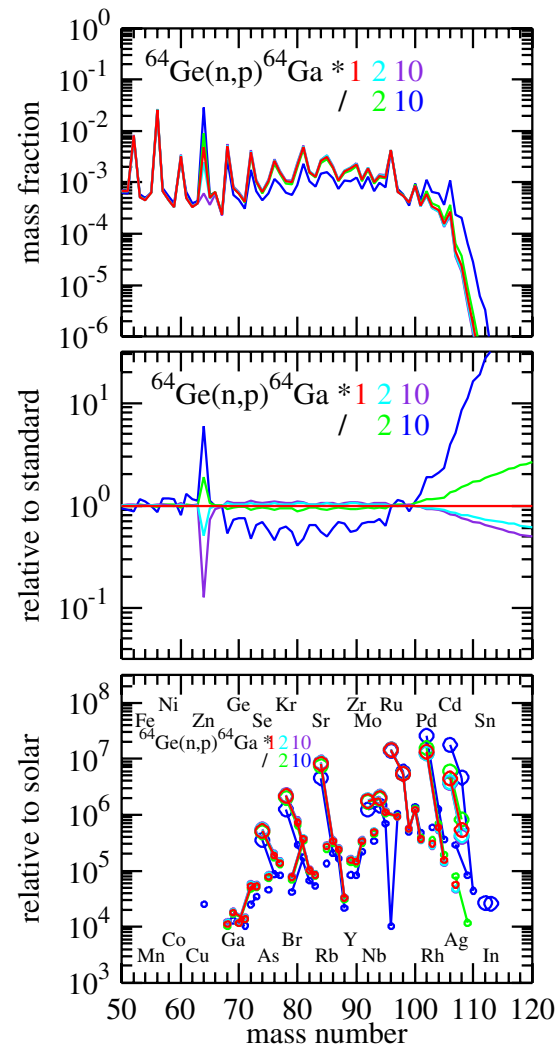

Figure 20. Same as Figure 2, but for variations on the ${ }^{64} \mathrm{Ge}(n, p){ }^{64} \mathrm{Ga}$ rate. (A color version of this figure is available in the online journal.)

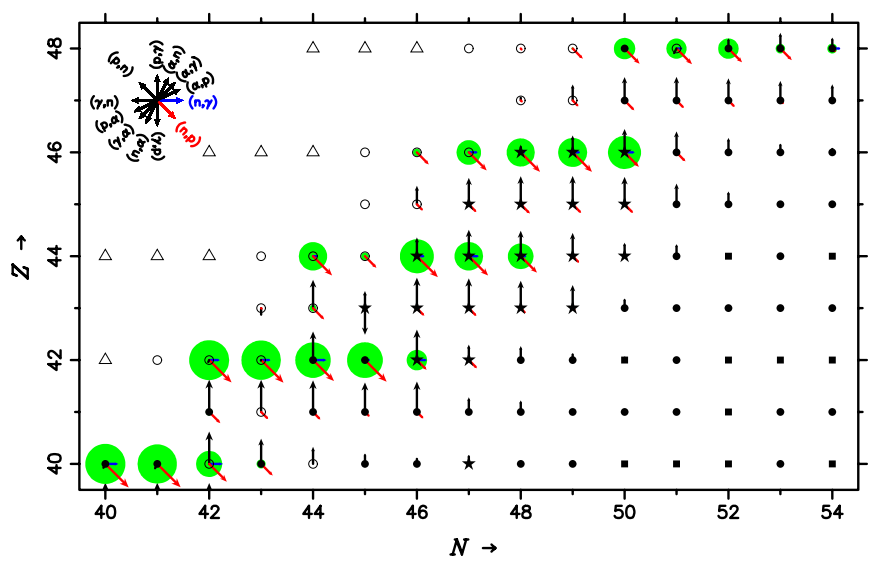

Figure 21. Same as Figure 17, but for the standard model with the new experimental masses of Weber et al. (2008, stars).

(A color version of this figure is available in the online journal.)

(first line in Tables 1 and 3). We confirm the suppression of the flow through ${ }^{87} \operatorname{Mo}(p, \gamma){ }^{88} \mathrm{Tc}(N=45$; see Figure 17$)$, which has been reported in Weber et al. (2008). This leads to a factor of three enhancement of ${ }^{87} \mathrm{Sr}$ and a factor of two reduction of ${ }^{89} \mathrm{Y}$, which are however not $p$-isotopes (and with small production factors). The other $p$-abundances, including ${ }^{92,94} \mathrm{Mo}$, are almost unchanged, as reported in Weber et al. (2008) and Fisker et al. (2009).

Our calculations of sensitivity tests for all other unmeasured masses on the $v p$-process path show that the mass of ${ }^{82} \mathrm{Zr}$ (or the proton separation energy of ${ }^{83} \mathrm{Nb}$ ) on $N=42$ plays an important role for production of a light $p$-nuclei ${ }^{84} \mathrm{Sr}$. The others have only minor roles for the sensitivity tests with variations of 


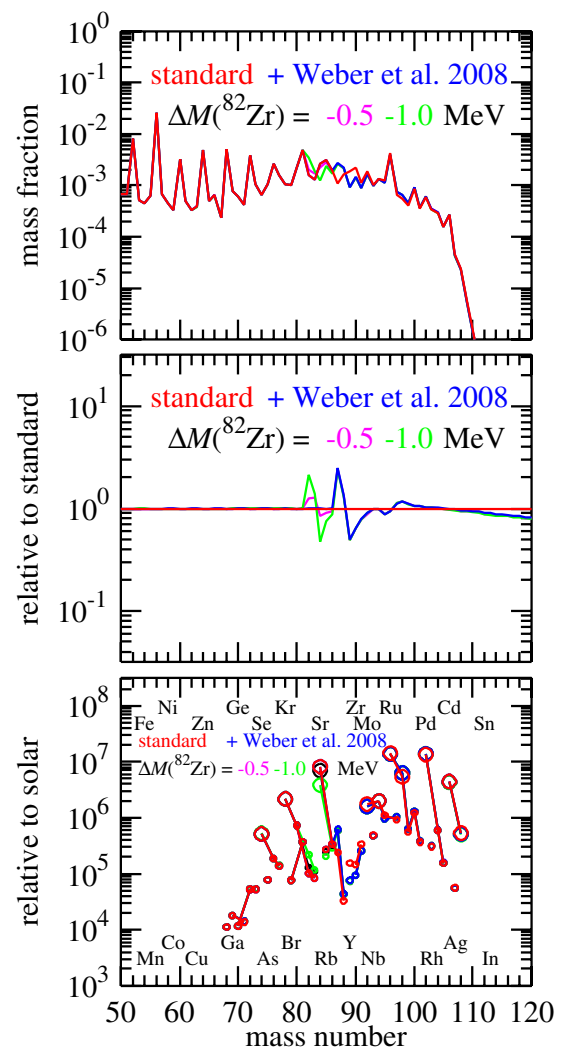

Figure 22. Same as Figure 2, but for the standard model (red) with the new experimental masses of Weber et al. (2008, blue). Also shown are the calculations with reductions $(-0.5$ and $-1.0 \mathrm{MeV})$ of the ${ }^{82} \mathrm{Zr}$ mass.

(A color version of this figure is available in the online journal.)

up to $\pm 1 \mathrm{MeV}$ on the nuclear masses. We find in Figure 22 that a $1.0 \mathrm{MeV}$ reduction of the ${ }^{82} \mathrm{Zr}$ mass (equivalent to a reduction of the proton separation energy of ${ }^{83} \mathrm{Nb}$ ) leads to a reduction of the ${ }^{84} \mathrm{Sr}$ abundance by a factor of two (middle panel). An increase of the ${ }^{82} \mathrm{Zr}$ mass has no effect on the $p$-abundances.

This is particularly important when we consider the role of the $v p$-process to the solar inventory of most mysterious $p$-nuclei, ${ }^{92}$ Mo and ${ }^{94}$ Mo. As can be seen in Figure 22 (bottom panel, and in other similar figures), the production factors of ${ }^{92} \mathrm{Mo}$ and ${ }^{94}$ Mo are always substantially smaller than the neighboring $p$-isotopes, in particular, ${ }^{84} \mathrm{Sr}$ (see Figures 6 and 7). A reduction of the ${ }^{84} \mathrm{Sr}$ would in part reduce this large gap. Note that the experimental mass of ${ }^{93} \mathrm{Nb}$ in Audi et al. (2003) involves a large uncertainty (315 keV). Future precision measurements of both ${ }^{82} \mathrm{Zr}$ and ${ }^{83} \mathrm{Nb}$ are thus highly desired.

\section{6. $v p$-PROCESS AS THE ORIGIN OF $p$-NUCLEI}

In the previous sections (Sections 4 and 5), we find that uncertainties in both the supernova dynamics and nuclear reactions can substantially affect the productivity of $p$-nuclei. This makes it difficult to determine the role of the $v p$-process as the source of the solar $p$-nuclei. Keeping such uncertainties in mind, we discuss a possible contribution of the $v p$-process to the solar $p$-abundances based on our result by comparing with other possible sources.

Table 4 lists the currently proposed astrophysical origins for each $p$-nuclide (first column) with its solar abundance and fraction relative to its elemental abundance (second and third columns; Lodders 2003). All these sources are associated with core-collapse supernovae. Photodissociation of pre- existing neutron-rich abundances in the oxygen-neon layer of core-collapse supernovae (or in their pre-collapse phases), i.e., the $\gamma$-process (Woosley \& Howard 1978; Prantzos et al. 1990; Rayet et al. 1995; Rauscher et al. 2002; Hayakawa et al. 2008) is currently regarded as the most successful scenario. In the fourth column of Table 4 , the $p$-nuclei whose origins can be explained by the $\gamma$-process in Rayet et al. (1995) are specified by "yes." The bracketed ones are those underproduced in a more recent work by Rauscher et al. (2002). The origins of up to 24 out of $35 p$-isotopes can be explained by the $\gamma$-process. However, the light $p$-isotopes $\left({ }^{92,94} \mathrm{Mo},{ }^{96,98} \mathrm{Ru},{ }^{102} \mathrm{Pd},{ }^{106,108} \mathrm{Cd}\right.$, ${ }^{113} \mathrm{In}$, and ${ }^{115} \mathrm{Sn}$ ), which account for a large fraction in the solar $p$-abundances, and some heavy $p$-isotopes $\left({ }^{138} \mathrm{La}\right.$ and $\left.{ }^{152} \mathrm{Gd}\right)$ need other sources (specified by "no" in Table 4).

The $v$-process (fifth column in Table 4; Woosley et al. 1990) in core-collapse supernovae is suggested to account for the production of a couple of heavy $p$-isotopes ${ }^{138} \mathrm{La}$ and ${ }^{180} \mathrm{Ta}$ (the former is underproduced in the $\gamma$-process). The $\alpha$-rich and slightly neutron-rich $\left(Y_{\mathrm{e}} \approx 0.47-0.49\right.$; slightly more protonrich than the $\beta$-stability values) neutrino-driven outflows were also suggested as the production site of some light $p$-isotopes including ${ }^{92}$ Mo (but not ${ }^{94}$ Mo; Hoffman et al. 1996; Wanajo 2006; Wanajo et al. 2009). The proton richness relative to the $\beta$-stability line in the fragmented QSE clusters (Hoffman et al. 1996; Meyer et al. 1998a) at $T_{9} \sim 4-3$ leads to the formation of these $p$-nuclei with $N \leqslant 50$. Such QSE clusters on the protonrich side of the $\beta$-stability line will be denoted as " $p$-QSE," hereafter. A recent study of nucleosynthesis in the electroncapture supernovae of a $9 M_{\odot}$ star shows that the lightest $p$-nuclei ${ }^{74} \mathrm{Se},{ }^{78} \mathrm{Kr},{ }^{84} \mathrm{Sr}$, and ${ }^{92} \mathrm{Mo}$ can be produced in $p$-QSE enough to account for their solar amounts (sixth column in Table 4; Wanajo et al. 2009). However, these additional sources still cannot fill the gap for some light $p$-isotopes such as ${ }^{94} \mathrm{Mo}$, ${ }^{96,98} \mathrm{Ru},{ }^{102} \mathrm{Pd},{ }^{106,108} \mathrm{Cd},{ }^{113} \mathrm{In},{ }^{115} \mathrm{Sn}$, and for a heavy $p$-isotope ${ }^{152} \mathrm{Gd}$.

Our result in this study is based on a semi-analytic model of neutrino-driven winds, while the results for the $\gamma$-process, the $\nu$-process, and the $p$-QSE listed in Table 4 are all based on realistic hydrodynamic studies. Nevertheless, we attempt to present a list of the $p$-isotopes whose origin can be attributed to the $v p$-process, as follows. The requisite overproduction factor for a given nuclide per supernova event, which explains its solar origin, is inferred to be $>10$ (e.g., Woosley et al. 1994). Assuming the masses of the total ejecta and of the neutrinodriven ejecta to be $\sim 10 M_{\odot}$ and $\sim 10^{-3} M_{\odot}$ (e.g., Wanajo 2006), the overproduction factor per supernova event is diluted by about four orders of magnitude compared with our result. We thus apply the condition $f>10^{5}$ and $f>f_{\max } / 10$ to each $p$ isotope abundance in Figure 6 (the standard model with $Y_{\mathrm{e}, 3}$ ranging between 0.5 and 0.7 ).

The $p$-isotopes that satisfy the above condition are listed in the last column of Table 4. According to recent hydrodynamic studies (Fischer et al. 2010; Hüdepohl et al. 2010), the maximum $Y_{\mathrm{e}}$ in the neutrino-driven outflows is $\sim 0.6$. Therefore, the $p$ isotopes that satisfy the above condition only with $Y_{\mathrm{e}, 3}>0.6$ are indicated by "[yes]." This implies that the $v p$-process in core-collapse supernovae is the possible astrophysical origin of the light $p$-nuclei up to $A=108$. In principle, however, the $v p$-process can account for the origin of the heavy $p$-isotopes up to $A=152$ as well, if $Y_{\mathrm{e}, 3} \approx 0.65$ (Figure 6) is achieved in the neutrino-driven outflows. If this is true, a reasonable combination of the astrophysical sources considered here can explain all the origins of the solar $p$-isotopes. It should be noted 
Table 4

p-Nuclei Abundances and Their Possible Sources

\begin{tabular}{|c|c|c|c|c|c|c|}
\hline Species & Abundance $^{\mathrm{a}}$ & Fraction $^{\mathrm{b}}(\%)$ & $\gamma$-Process ${ }^{\mathrm{c}}$ & $\nu$-Process ${ }^{\mathrm{d}}$ & $p$-QSE ${ }^{\mathrm{e}}$ & $v p$-Process $\mathrm{f}$ \\
\hline${ }^{74} \mathrm{Se}$ & 0.58 & 0.889 & Yes & No & Yes & Yes \\
\hline${ }^{78} \mathrm{Kr}$ & 0.20 & 0.362 & Yes & No & Yes & Yes \\
\hline${ }^{84} \mathrm{Sr}$ & 0.13124 & 0.5551 & Yes & No & Yes & Yes \\
\hline${ }^{92} \mathrm{Mo}$ & 0.386 & 14.8362 & No & No & Yes & Yes \\
\hline${ }^{94} \mathrm{Mo}$ & 0.241 & 9.2466 & No & No & No & Yes \\
\hline${ }^{96} \mathrm{Ru}$ & 0.1053 & 5.542 & No & No & No & Yes \\
\hline${ }^{98} \mathrm{Ru}$ & 0.0355 & 1.8688 & No & No & No & Yes \\
\hline${ }^{102} \mathrm{Pd}$ & 0.0146 & 1.02 & No & No & No & Yes \\
\hline${ }^{106} \mathrm{Cd}$ & 0.01980 & 1.25 & No & No & No & Yes \\
\hline${ }^{108} \mathrm{Cd}$ & 0.01410 & 0.89 & No & No & No & Yes \\
\hline${ }^{113} \mathrm{In}$ & 0.0078 & 4.288 & No & No & No & [Yes] \\
\hline${ }^{112} \mathrm{Sn}$ & 0.03625 & 0.971 & [Yes] & No & No & [Yes] \\
\hline${ }^{114} \mathrm{Sn}$ & 0.02460 & 0.659 & [Yes] & No & No & [Yes] \\
\hline${ }^{115} \mathrm{Sn}$ & 0.01265 & 0.339 & No & No & No & [Yes] \\
\hline${ }^{120} \mathrm{Te}$ & 0.0046 & 0.096 & [Yes] & No & No & [Yes] \\
\hline${ }^{124} \mathrm{Xe}$ & 0.00694 & 0.129 & [Yes] & No & No & [Yes] \\
\hline${ }^{126} \mathrm{Xe}$ & 0.00602 & 0.112 & Yes & No & No & [Yes] \\
\hline${ }^{130} \mathrm{Ba}$ & 0.00460 & 0.1058 & Yes & No & No & [Yes] \\
\hline${ }^{132} \mathrm{Ba}$ & 0.00440 & 0.1012 & Yes & No & No & [Yes] \\
\hline${ }^{138} \mathrm{La}$ & 0.000397 & 0.09017 & No & Yes & No & [Yes] \\
\hline${ }^{136} \mathrm{Ce}$ & 0.00217 & 0.186 & Yes & No & No & [Yes] \\
\hline${ }^{138} \mathrm{Ce}$ & 0.00293 & 0.251 & Yes & No & No & [Yes] \\
\hline${ }^{144} \mathrm{Sm}$ & 0.00781 & 3.0734 & Yes & No & No & [Yes] \\
\hline${ }^{152} \mathrm{Gd}$ & 0.00067 & 0.2029 & No & No & No & [Yes] \\
\hline${ }^{156} \mathrm{Dy}$ & 0.000216 & 0.056 & [Yes] & No & No & No \\
\hline${ }^{158}$ Dy & 0.000371 & 0.096 & [Yes] & No & No & No \\
\hline${ }^{162} \mathrm{Er}$ & 0.000350 & 0.137 & [Yes] & No & No & No \\
\hline${ }^{164} \mathrm{Er}$ & 0.004109 & 1.609 & [Yes] & No & No & No \\
\hline${ }^{168} \mathrm{Yb}$ & 0.000323 & 0.13 & Yes & No & No & No \\
\hline${ }^{174} \mathrm{Hf}$ & 0.000275 & 0.1620 & Yes & No & No & No \\
\hline${ }^{180} \mathrm{Ta}$ & 0.00000258 & 0.0123 & Yes & Yes & No & No \\
\hline${ }^{180} \mathrm{~W}$ & 0.000153 & 0.1198 & Yes & No & No & No \\
\hline${ }^{184} \mathrm{Os}$ & 0.000133 & 0.0198 & Yes & No & No & No \\
\hline${ }^{190} \mathrm{Pt}$ & 0.000185 & 0.013634 & Yes & No & No & No \\
\hline${ }^{196} \mathrm{Hg}$ & 0.00063 & 0.15344 & Yes & No & No & No \\
\hline
\end{tabular}

Notes.

${ }^{a}$ Lodders (2003); $\mathrm{Si}=10^{6}$.

b Lodders (2003); relative to its elemental abundance.

c Rayet et al. (1995) (nuclei indicated by "[yes]" are those underproduced in Rauscher et al. 2002).

d Woosley et al. (1990).

e Wanajo et al. (2009).

f This work (nuclei indicated by "[yes]" are produced only with $Y_{\mathrm{e}, 3}>0.6$ ).

that most of the maximum production factors of these heavy $p$-nuclei are $\gtrsim 10^{8}$. This is three orders of magnitude larger than the above requisite value $\left(f=10^{5}\right)$. Thus, only $\sim 0.1 \%$ of neutrino-driven ejecta with $Y_{\mathrm{e}, 3} \approx 0.60-0.65$ is enough to account for the origin of these heavy $p$-nuclei. Future multidimensional hydrodynamic studies of core-collapse supernovae with full neutrino transport will be of particular importance if such a condition is indeed obtained.

A word of caution for the molybdenum isotopes is needed here. The production factors of ${ }^{92}$ Mo and ${ }^{94}$ Mo satisfy the above condition only marginally with $Y_{\mathrm{e}, 3}=0.53-0.54$. The future measurements of the nuclear masses of ${ }^{82} \mathrm{Zr}$ and ${ }^{83} \mathrm{Nb}$ might in part cure this problem as discussed in Section 5.3. This is rather serious for the origin of ${ }^{94} \mathrm{Mo}$ that can be produced only by the $v p$-process, while ${ }^{92} \mathrm{Mo}$ can be explained by the $p$-QSE. Fisker et al. (2009) concluded that the ratio ${ }^{92} \mathrm{Mo} /{ }^{94} \mathrm{Mo}$ is about five times smaller than the solar value, when applying the proton separation energy of ${ }^{93} \mathrm{Rh}$ in Weber et al. (2008). This might imply that ${ }^{92}$ Mo has another origin, presumably the $p$-QSE. We however obtain a reasonable ratio with our standard model (see, e.g., the bottom panel of Figure 22) and many other cases (see the $Y_{\mathrm{e}, 3} \leqslant 0.55$ range in Figure 6). This is due to the significant role of ${ }^{92} \mathrm{Ru}(n, p){ }^{92} \mathrm{Tc}$ that competes with ${ }^{92} \mathrm{Ru}(p, \gamma){ }^{93} \mathrm{Rh}$ in our cases. This is a consequence of the values of $\Delta_{n}$ in the present cases being about a factor of three higher than those in Pruet et al. (2006). This suggests that ${ }^{92} \mathrm{Mo} /{ }^{94} \mathrm{Mo}$ is highly sensitive to the details of supernova dynamics.

\section{SUMMARY}

We investigated the effects of uncertainties in supernova dynamics as well as in nuclear data inputs on the $v p$-process in the neutrino-driven outflows of core-collapse supernovae. The former includes the wind-termination radius $r_{\mathrm{wt}}$ (or temperature $T_{\mathrm{wt}}$ ), neutrino luminosity $L_{v}$, neutron-star mass $M_{\mathrm{ns}}$, and electron fraction $Y_{\mathrm{e}, 9}$ (or $Y_{\mathrm{e}, 3}$, at $T_{9}=9$ and 3, respectively). The latter includes the reactions relevant to the breakout from the $p-p$ chain region $(A<12)$, the $(n, p)$ reactions on heavy 
nuclei $(Z \geqslant 56)$, and the nuclear masses $(40 \leqslant Z \leqslant 50)$ on the $v p$-process pathway. Our result is summarized as follows.

1. Wind termination of the neutrino-driven outflow by colliding with the preceding supernova ejecta causes a slowdown of the temperature decrease and thus plays a crucial role on the $v p$-process. The termination within the temperature range of $T_{9}=1.5-3$ (relevant to the $v p$-process) substantially increases the number of neutrons captured by the seed nuclei $\left(\Delta_{\mathrm{n}}\right)$ and thus enhances efficiency of the $p$-nuclei production. In the current case, the efficiency is maximal at $T_{\mathrm{wt}, 9}=2.65\left(r_{\mathrm{wt}}=231 \mathrm{~km}\right.$ for $\left.L_{v}=10^{52} \mathrm{erg} \mathrm{s}^{-1}\right)$. This implies that the early wind phase with the termination radius close to the proto-neutron star surface is favored for the $v p$-process.

2. A lower $L_{v}$ (with the other parameters $T_{\mathrm{wt}}, M_{\mathrm{ns}}$, and $Y_{\mathrm{e}, 9}$ unchanged) leads to more efficient $v p$-processing. This is due to the larger entropy per nucleon for a lower $L_{v}$, which increases the proton-to-seed ratio $Y_{\mathrm{p}} / Y_{\mathrm{h}}$ and thus $\Delta_{\mathrm{n}}$. However, the role of the wind termination is more crucial and thus we presume that the maximum efficiency is obtained during the early phase with $L_{v} \sim 10^{52} \mathrm{erg} \mathrm{s}^{-1}$.

3. A larger $M_{\mathrm{ns}}$ (with the other parameters $T_{\mathrm{wt}}, L_{v}$, and $Y_{\mathrm{e}, 9}$ unchanged) results in a larger efficiency of the $v p$-process. This is a consequence of the larger entropy per nucleon and the faster expansion of the neutrino-driven outflow for a larger $M_{\mathrm{ns}}$, both of which help to increase $Y_{\mathrm{p}} / Y_{\mathrm{h}}$ and thus $\Delta_{\mathrm{n}}$. This implies that a more massive progenitor is favored for more efficient $v p$-processing, if other parameters remain unchanged. In reality, however, the evolutions of $L_{v}, r_{\mathrm{wt}}$, and $Y_{\mathrm{e}}$ will be dependent on the progenitor mass, making it difficult to draw definitive conclusions.

4. The $v p$-process is highly sensitive to the electron fraction $Y_{\mathrm{e}, 3}$ that controls $Y_{\mathrm{p}} / Y_{\mathrm{h}}$ at the onset of the $v p$-process and thus $\Delta_{\mathrm{n}}$. An increase of only $\Delta Y_{\mathrm{e}, 3} \sim 0.03$ results in $\Delta A_{\max } \sim 10$. The models with $Y_{\mathrm{e}, 3}=0.52-0.60$ (with the other parameters unchanged) produce sufficient amounts of the light $p$-nuclei up to $A=108$. Furthermore, the models with $Y_{\mathrm{e}, 3}=0.60-0.65$ produce the $p$-nuclei up to $A=$ 152. Note that this is a combined effect of the high $Y_{\mathrm{e}, 3}$ and the wind termination at sufficiently high temperature ( $T_{\mathrm{wt}, 9}=2.19$ in the standard model). Our result shows no substantial enhancement of the $p$-nuclei with $A>152$, since the nuclear flow reaches the $\beta$-stability line and enters to the neutron-rich region at $A \sim 130-160$. This is a consequence that a large $\Delta_{\mathrm{n}}$ leads to the strong $(n, \gamma)$ flows that compete with those by $(p, \gamma)$ for $Z>50$.

5. Variations on the nuclear reactions relevant to the breakout from the $p-p$ chain region $(A<12)$, namely, of triple- $\alpha$, ${ }^{7} \mathrm{Be}(\alpha, \gamma){ }^{11} \mathrm{C}$, and ${ }^{10} \mathrm{~B}(\alpha, p){ }^{13} \mathrm{C}$ affect the $v p$-process by changing $Y_{\mathrm{p}} / Y_{\mathrm{h}}$ (and $\Delta_{\mathrm{n}}$ ) or producing intermediate-mass nuclei (proton poison) during $v p$-processing. Among these reactions, triple- $\alpha$ has the largest impact, although the other two show non-negligible effects, on the production of the $p$-nuclei at $A \sim 100-110$.

6. Variations on the $(n, p)$ reactions on ${ }^{56} \mathrm{Ni}$ (seed nuclei), ${ }^{60} \mathrm{Zn}$, and ${ }^{64} \mathrm{Ge}$ (first $\beta^{+}$-waiting point on the classical rp-process) show great impact on efficiency of the $v p$ process for heavy element synthesis. Only a factor of two variation leads to a factor of 10 or more changes in the production of the $p$-nuclei with $A \sim 100-110$ for the first reaction (but somewhat smaller changes for the latter two reactions). This is a consequence of the fact that these reactions control the strength of the nuclear flow passing through the ( $n, p)$-waiting points $\left({ }^{56} \mathrm{Ni},{ }^{60} \mathrm{Zn}\right.$, and $\left.{ }^{64} \mathrm{Ge}\right)$ on the $v p$-process path. We also find that the $N=50$ nucleus ${ }^{96} \mathrm{Pd}$ plays a role of the "second seed" for production of heavier nuclei.

7. Application of the new experimental masses of Weber et al. (2008; for $39 \leqslant Z \leqslant 46$ ) exhibits a suppression of the flow ${ }^{87} \operatorname{Mo}(p, \gamma){ }^{88} \mathrm{Tc}(N=45)$, which, however, does not affect the nucleosynthetic $p$-abundances. Our sensitivity tests for unmeasured nuclear masses indicate that a future measurement of the ${ }^{82} \mathrm{Zr}$ mass (and of ${ }^{93} \mathrm{Nb}$ with a large estimated error) on $N=42$ could reduce the abundance of ${ }^{84} \mathrm{Sr}$ by a factor of two.

8. Our result implies that, within possible ranges of uncertainties in supernova dynamics as well as in nuclear data inputs, the solar inventory of the light $p$-nuclei up to $A=$ $108\left({ }^{108} \mathrm{Cd}\right)$ can be attributed to the $v p$-process, including the most mysterious ones, ${ }^{92,94} \mathrm{Mo}$ and ${ }^{96,98} \mathrm{Ru}$. The molybdenum isotopes, however, tend to be underproduced compared with the neighboring $p$-isotopes. If highly proton-rich conditions with $Y_{\mathrm{e}, 3}=0.60-0.65$ are realized in neutrinodriven ejecta, the solar origin of the $p$-nuclei up to $A=152$ $\left({ }^{152} \mathrm{Gd}\right)$ can be explained by the $v p$-process.

Our explorations in this study suggest that more refinements both in supernova conditions and in nuclear data inputs are needed to elucidate the role of the $v p$-process as the astrophysical origin of the $p$-nuclei. In particular, multi-dimensional studies of core-collapse simulations with full neutrino transport, as well as experiment-based rates of triple- $\alpha$ and the $(n, p)$ reactions on heavy nuclei will be important in the future works.

We are grateful to T. Shima for useful discussion on the triple$\alpha$ rate. The project was supported by the Deutsche Forschungsgemeinschaft through Cluster of Excellence EXC 153 "Origin and Structure of the Universe" (http://www.universe-cluster.de).

\section{REFERENCES}

Aikawa, M., Arnould, M., Goriely, S., Jorissen, A., \& Takahashi, K. 2005, A\&A, 441, 1195

Angulo, C., et al. 1999, Nucl. Phys. A, 656, 3

Arcones, A., Janka, H.-Th., \& Scheck, L. 2006, A\&A, 467, 1227

Audi, G., Wapstra, A. H., \& Thibault, C. 2003, Nucl. Phys. A, 729, 337

Austin, S. M. 2005, Nucl. Phys. A, 758, 375

Brown, B. A., Clement, R. R., Schatz, H., Volya, A., \& Richter, W. A. 2002, Phys. Rev. C, 65, 045802

Buras, R., Rampp, M., Janka, H.-Th, \& Kifonidis, K. 2006, A\&A, 447, 1049

Burrows, A., Hayes, J., \& Fryxell, B. A. 1995, ApJ, 450, 830

Caughlan, G. R., \& Fowler, W. A. 1988, At. Data Nucl. Data Tables, 40, 283

Diget, C. Aa., et al. 2005, Nucl. Phys. A, 760, 3

Diget, C. Aa., et al. 2009, Phys. Rev. C, 80, 034316

Fischer, T., Whitehouse, S. C., Mezzacappa, A., Thielemann, F.-K., \& Liebendörfer, M. 2010, A\&A, 517, 80

Fisker, J. L., Hoffman, R. D., \& Pruet, J. 2009, ApJ, 690, L135

Fröhlich, C., et al. 2006a, ApJ, 637, 415

Fröhlich, C., et al. 2006b, Phys. Rev. Lett., 96, 142502

Fuller, G. M., Fowler, W. A., \& Newman, M. J. 1982, ApJS, 48, 279

Goriely, S., Samyn, M., Pearson, J. M., \& Onsi, M. 2005, Nucl. Phys. A, 750, 425

Hayakawa, T., Iwamoto, N., Kajino, T., Shizuma, T., Umeda, H., \& Nomoto, K. 2008, ApJ, 685, 1089

Hayakawa, S., et al. 2010, in AIP Conf. Proc. 1269, 10th International Symposium on Origin of Matter and Evolution of Galaxies, ed. I. Tanihata et al. (OMEG2010) (Melville, NY: AIP), 104

Hoffman, R. D., Woosley, S. E., Fuller, G. M., \& Meyer, B. S. 1996, ApJ, 460, 478

Hüdepohl, L., Müller, B., Janka, H.-Th., Marek, A., \& Raffelt, G. G 2010, Phys. Rev. Lett., 104, 251101

Janka, H.-Th., Buras, R., \& Rampp, M. 2003, Nucl. Phys. A, 718, 269 
Janka, H.-T., \& Müller, E. 1995, ApJ, 448, L109

Janka, H.-T., \& Müller, E. 1996, A\&A, 306, 167

Kitaura, F. S., Janka, H.-Th., \& Hillebrandt, W. 2006, A\&A, 450, 345

Kuroda, T., Wanajo, S., \& Nomoto, K. 2008, ApJ, 672, 1068

Langanke, K., \& Martinez-Pinedo, G. 2001, At. Data Nucl. Data Tables, 79, 1

Liebendörfer, M., Mezzacappa, A., Messer, O. E. B., Martinez-Pinedo, G., Hix, W. R., \& Thielemann, F.-K. 2003, Nucl. Phys. A, 719, 144

Lodders, K. 2003, ApJ, 591, 1220

McLaughlin, G. C., Fuller, G. M., \& Wilson, J. R. 1996, ApJ, 472, 440

Meyer, B. S., Krishnan, T. D., \& Clayton, D. D. 1998a, ApJ, 498, 808

Meyer, B. S., McLaughlin, G. C., \& Fuller, G. M. 1998b, Phys. Rev. C, 58, 3696

Müller, B., Janka, H.-Th., \& Dimmelmeier, H. 2010, ApJS, 189, 104

Otsuki, K., Tagoshi, H., Kajino, T., \& Wanajo, S. 2000, ApJ, 533, 424

Panov, I. V., \& Janka, H.-Th. 2009, A\&A, 494, 829

Prantzos, N., Hashimoto, M., Rayet, M., \& Arnould, M. 1990, A\&A, 238, 455

Pruet, J., Hoffman, R. D., Woosley, S. E., Buras, R., \& Janka, H.-Th. 2006, ApJ, 644, 1028

Qian, Y. -Z., \& Woosley, S. E. 1996, ApJ, 471, 331

Rauscher, T. 2010, Phys. Rev. C, 81, 045807

Rauscher, T., Heger, A., Hoffman, R. D., \& Woosley, S. E. 2002, ApJ, 576, 323
Rauscher, T., Thielemann, F.-K., \& Kratz, K.-L. 1997, Phys. Rev. C, 56, 1613 Rayet, M., Arnould, M., Hashimoto, M., Prantzos, N., \& Nomoto, K. 1995, A\&A, 298, 517

Schatz, H., et al. 2001, Phys. Rev. Lett., 86, 3471

Seitenzahl, I. R., Timmes, F. X., Marin-Lafleche, A., Brown, E., Magkotsios, G., \& Truran, J. 2008, ApJ, 685, L129

Tachibana, T., Yamada, M., \& Yoshida, Y. 1990, Prog. Theor. Phys., 84, 641

Timmes, F. X., \& Swesty, F. D. 2000, ApJS, 126, 501

Wagoner, R. V. 1969, ApJS, 18, 247

Wallace, R. K., \& Woosley, S. E. 1981, ApJS, 45, 389

Wanajo, S. 2006, ApJ, 647, 1323

Wanajo, S. 2007, ApJ, 666, L77

Wanajo, S., Kajino, T., Mathews, G. J., \& Otsuki, K. 2001, ApJ, 554, 578

Wanajo, S., Nomoto, K., Janka, H.-T., Kitaura, F. S., \& Müller, B. 2009, ApJ, 695, 208

Weber, C., et al. 2008, Phys. Rev. C, 78, 054310

Woosley, S. E., Hartmann, D. H., Hoffman, R. D., \& Haxton, W. C. 1990, ApJ, 356,272

Woosley, S. E., \& Howard, W. M. 1978, ApJS, 36, 285

Woosley, S. E., Wilson, J. R., Mathews, G. J., Hoffman, R. D., \& Meyer, B. S. 1994, ApJ, 433, 229 